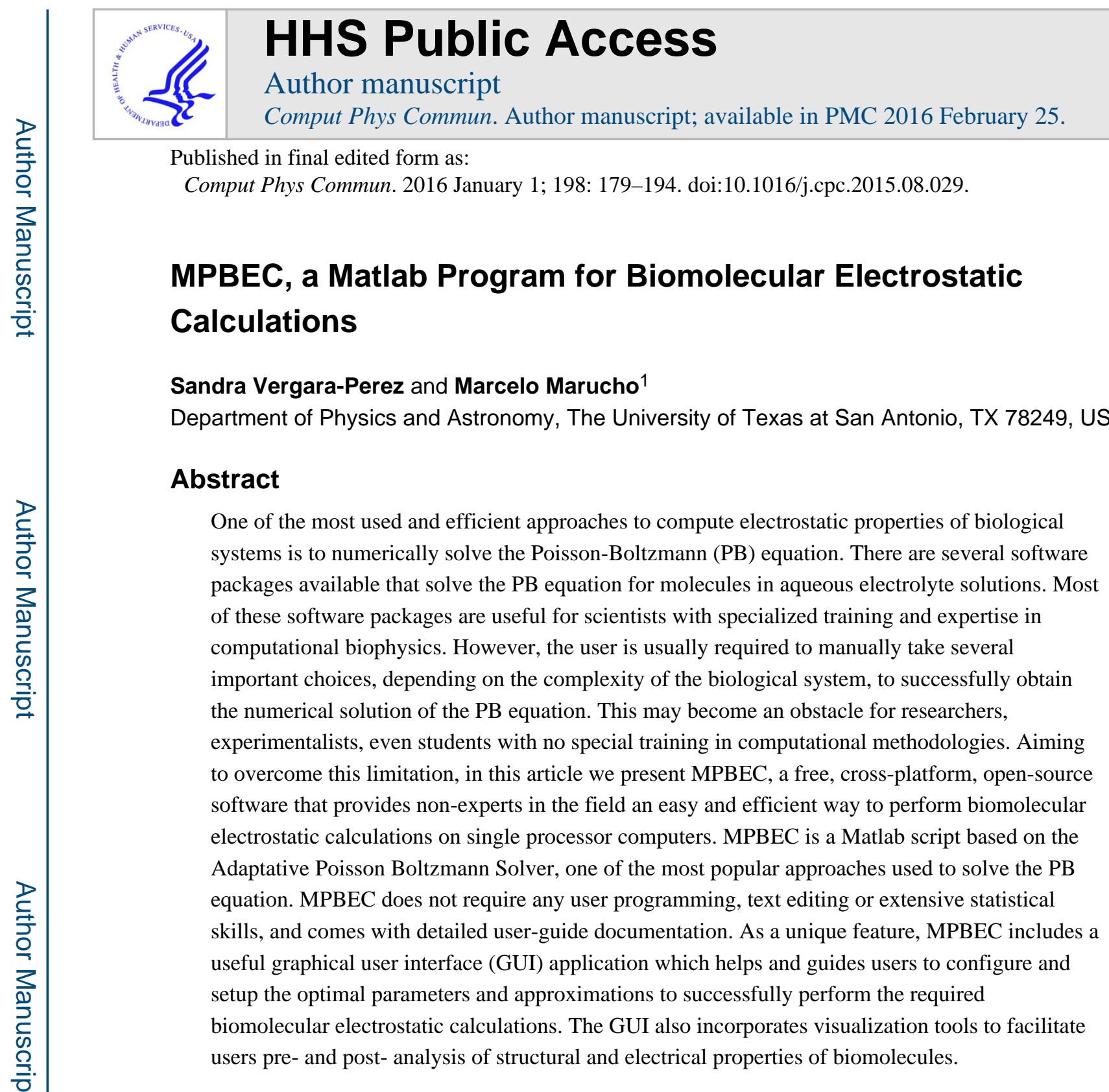

\title{
Keywords
}

Poisson-Boltzmann equation; biomolecular electrostatic calculations; mean electrostatic potential; Matlab software; Graphical user interface

\section{Program Summary}

Program Title: MPBEC

Journal Reference:

Catalogue identifier:

\footnotetext{
${ }^{1}$ Corresponding author: marcelo.marucho@utsa.edu.
} 
Program obtainable from: CPC Program Library; The University at Texas at San Antonio, TX, USA.

Licensing provisions: UTSA license.

Distribution format: zip

Programming language: Matlab R2010a and higher versions.

Computer: any computer at least with $2.5 \mathrm{GHz}$ speed.

Operating system: Windows, Linux Fedora, and Mac OSX.

RAM: at least $2 \mathrm{~GB}$ of free memory.

Classification: $3,10$.

External routines/libraries: Java.

Subprograms used: Jmol, pdb2pqr, Propka.

Nature of problem: Numerical solution of the linearized PB equation.

Solution method: MPBEC uses sparse matrix calculations and iterative linear solvers (gmres, minres, and bicgstab) to solve the discretized (box method) linear PB equation. The stability and the convergence of these iterative solvers is improved by using the incomplete LU factorization which generates pre-conditioner matrices $L$ (lower triangular matrix) and $U$ (upper triangular matrix) at low computational cost.

Unusual features: MPBEC incorporates a GUI to provide non-experts in computational biophysics an user-friendly and intuitive tool to obtain biomolecular electrostatic calculations. The GUI provides helpful information about how to fill out the input data by moving the mouse pointer over the corresponding text or blank box. The GUI tests all the input data before running MPBEC to make sure that the solver is properly configured and facilitates the user the pre- and post-analysis of the biomolecular calculations.

Additional comments: MPBEC checks available and required RAM memory before allocation to alert users when the available RAM memory is insufficient to perform the required calculations depending basically on the number of grid points and the size and number of atoms of the biomolecule(s). Therefore, MPBEC might be used with lower RAM memory and processor speed conditions than those mentioned above.

Running time: the software runs on single processor computers at low-to-moderate computational cost depending on the computer performance, the biomolecule size, the grid resolution, and required calculations. 


\section{Overview}

Electrostatics plays a fundamental role in many physical, chemical, and biological processes involving molecules in aqueous electrolyte solutions. The Poisson-Boltzmann (PB) equation constitutes one of the most used approaches to treat electrostatic effects in solution with a wide range of applications including the computation of the mean electrostatic potential $\Phi(\mathrm{r})$, the solvation free energy of molecules in solution, the free energy of association between solutes and ligands and its salt dependence, and the study of $\mathrm{pH}$ effects on these properties [1]. In many biologically relevant systems the solution of the (approximate) linear PB equation is close to that one obtained from the (exact) nonlinear PB equation, even when the linear approximation does not hold. Additionally, the numerical calculation of the former is much more efficient that the later one, making the linearized PB equation the most popular approach for biomolecular electrostatic calculations. The linearized PB equation is given by the following expression

$$
-\nabla \cdot(\epsilon(\mathbf{r}) \nabla u(\mathbf{r}))+\bar{k}^{2}(\mathbf{r}) u(\mathbf{r})=\frac{4 \pi e_{c}^{2}}{K_{B} T} \sum_{i=1}^{N} z_{i} \delta\left(\mathbf{r}-\mathbf{r}_{i}\right)
$$

where $u(\mathbf{r})=e_{C} \Phi(\mathbf{r}) / K_{B} T$ is the dimensionless electric potential, $\Phi(\mathrm{r})$, the mean electrostatic potential, $\varepsilon(r)$, the dielectric permittivity tensor, $K_{B}$, the Boltzmann constant, $T$, the absolute temperature, $e_{c}$, the elementary charge, $\bar{\kappa}$, the Debye-Hückel function (related to the ionic strength), and $z$ is the charge valence.

There are several approaches proposed to obtain the numerical solution of the Eq. (1) including finite difference, finite element, and boundary element [2]. The numerical solution is usually obtained by using high performance algorithms which discretize the differential operators, the dielectric tensor, the Debye-Hückel function, and the molecular charge distribution on a mesh of grid points. Then, the solvent accessible molecular surface is calculated and the resulting linear matrix equation for the discretized mean electrostatic potential is solved. There is a variety of approximations and approaches that can be used to perform these calculations. Many of these algorithms are becoming part of the "tool kit" used by everyone who wish to obtain an understanding of the electrostatic properties of biological systems. While most of these software perform automatic calculations [3], the user is usually required to manually take several important choices to obtain the correct PB solution. In the early years of biomolecular electrostatic calculations, almost all scientists working in the field received specialized training that provided a detailed understanding of the power and limitations of the PB solvers. Nowadays, the methodology is becoming more accessible, in terms of availability of programs and required computational resources, and better validated, in terms of published results. As a consequence, interesting applications to important and complex biological systems are now often carried out by researchers, experimentalists, even students with no special training in computational methodologies.

In this article we introduce MPBEC (Matlab Program for Biomolecular Electrostatic Calculations), a free, cross-platform, open-source software that provides non-experts in the field an easy and efficient way to perform biomolecular electrostatic calculations. MPBEC is a MATLAB script based on the Adaptative Poisson Boltzmann Solver (APBS) [4], one of 
the most popular approaches used to solve the PB equation. MPBEC does not require user programming, text editing, or extensive statistical skills, and comes with detailed user-guide documentation. MPBEC takes advantage of optimized Matlab routines specially designed to solve large sparse linear system of equations. This feature enables the software to run on single processor computers at low-to-moderate computational cost depending on the computer performance, the biomolecule size, and the grid resolution, among other factors. More importantly, MPBEC includes a graphical user interface (GUI) (see Figure 1) that allows users to take advantage of the visually guided setup of the required input data to solve the linear PB equation. The GUI eliminates the laborious and error prone generation in the manual entry of data and substantially reduces the time spent on the setup of the input file. As a unique feature, the GUI provides helpful information about how to fill out the input data by moving the mouse pointer over the corresponding text or blank box (see Figure 2). The GUI tests all the input data before running MPBEC to make sure that the solver is properly configured for the required calculations. Consequently, the GUI does not run MPBEC if the pre-test algorithm detects any unusual/nonphysical value assigned to the required parameters as well as if any required input data is missing. Instead, the GUI sends the user warning messages to inform what is the issue(s) that must be fixed (see Figure 3). Additionally, before any calculation MPBEC determines the available RAM memory of the user computer and estimates the RAM memory required to perform the required calculations to avoid memory allocation issues. The GUI provides default values of key input parameters and preselects some relevant algorithms to speed up the setup of the input data. These predefined parameters and algorithms may be easily changed at any time if needed. The GUI also integrates a web browser to assist the user in obtaining molecular structures (pdb files) from the protein data bank website. In such a case, the GUI uses pdb2pqr, an open source application (http://sourceforge.net/projects/pdb2pqr/), to automatically convert the molecular structures into the format required to solve the PB equation (pqr files) [4]. Moreover, the GUI facilitates the user the pre- and post- analysis of the biomolecular electrostatic calculations. It integrates Jmol, an open-source Java viewer for chemical structures in 3D (http://www.jmol.org/), to analyze molecular structures (see Figure 4) and display mean electrostatic potential solutions of the PB equation at the solvent accessible surface of the biomolecule (see Figure 5). As a result, the GUI enables the user to visualize the charge distribution, size, and shape of molecules in 3D, which is of fundamental importance in determining how molecules interact with one another. Additionally, the GUI utilizes Matlab plot tools to visualize the surface mean electrostatic potential along the plane $z=L_{z} / 2$, where $L_{z}$ is the box size along the $z$ direction (see Figure 6). This visualization is useful for analyzing the behavior of the mean electrostatic potential at short and long distances from the biomolecule(s). It is also relevant for analyzing the effective range, intensity, and nature of the electrostatic interaction between the analyzed biomolecule(s) and surrounding ions. At the end of the calculations, MPBEC writes out a log file containing all information displayed on the Matlab console, including step by step intermediate calculations and computing times (see Figure 7). Additionally, all required output data files are properly saved and organized in a user-designated folder for post-analysis purposes. Finally, parallelization and implementation of MPBEC into existing Matlab programs is straightforward (more information is provided in Appendix A). 
In the next sections of this article we explain how to run and use the GUI. We also provide several examples to illustrate the use of the GUI and the solver performance. In Appendix A we provide a description on how to run MPBEC without the GUI whereas Appendix B includes a detailed description of the theoretical approach and computational scheme implemented by MPBEC to solve the linear PB equation.

\section{How to run the Graphical User Interface}

There are two ways to run the GUI. We recommend to use the provided batch files. For Windows users, just double click "mpbec-win.bat" to open the GUI automatically in Matlab console mode. On the other hand, Mac (Linux) users have to run the batch file "mpbecmac.sh" ("mpbec-lin.sh") from a terminal. For instance, if you are a Linux user and your / path/to/the/file is "/home/smith/Documents/MPBEC/mpbec-lin.sh", you have to run "source /home/smith/Documents/MPBEC/mpbec-lin.sh".

Mac (Linux) users may run "find -name mpbec-mac.sh 2>/dev/null" ("find /home -name mpbec-lin.sh 2>/dev/null”) to get the /path/to/the/file if needed.

Alternatively, the user can run Matlab as usual, change the current directory to " /MPBEC/ program/src/Main" using the Matlab search toolbar, and type "Welcome" in the Matlab window console. This GUI introduces MPBEC and provides the user the option to run the Beginner or Advanced level mode depending on the experience of the user performing biomolecular electrostatics calculations. In the Beginner mode, the user only has to upload the molecular structure file(s), choose, if needed, an energetic calculation option, provide a name for the input file, and run the calculation. MPBEC automatically assigns the remaining parameters. In the Advanced mode, the user has full access to the configuration of MPBEC.

Once MPBEC has finished all the calculations, the user may change the configuration of the GUI and run MPBEC again. If the user closes the GUI before all the calculations are finished, the current directory may not be the one required to run the GUI again. Make sure that the current directory in the Matlab console is " $/ \mathrm{MPBEC} / \mathrm{program} / \mathrm{src} /$ Main" before running the GUI. If any unexpected message/warning is received in the Matlab window console and the user is unable to run the GUI, the user must exit Matlab and start over again.

\section{How to use the Graphical User Interface}

In principle, there is no pre-established order to fill out boxes and select options in the GUI. However, it is highly recommended to provide the mandatory input data first, e.g. the "molecular structure" file(s), the "output directory", and the "input file name", and subsequently any other information if needed. Preselected options and predefined values presented in the main panel can be easily changed by the user at any time if needed. The user should be able to obtain the required PB solution without using optional panels. However, the user may need to provide additional information using optional panels depending on specific (advanced) calculations. After all changes on preselected parameters and options are completed, the user is able to run MPBEC by pressing the "Compute Solution" button. 
Below we explain how to change any preselected option and predefined value as well as how to setup optional panels.

\section{GUI description}

There are several panels in the GUI that help and guide the user to configure and setup all the required parameters to solve the linear PB equation (see Figure 1).

The "Charge map approx" and "Dielectric and kappa maps approx" panels (also referred as $\mathrm{chm}$ and $\mathrm{dkm}$, respectively) enable the user to select several options to calculate the charge density, dielectric, and kappa (ion-accessibility) maps. SPL2 and MOL, two of the most frequently used approximations, are preselected for the "Charge map approx" and "Dielectric and kappa maps approx" panels, respectively. Alternatively, the user can activate the "Map files (optional)" panel to upload map files in $\mathrm{dx}$ format. Clearly, the use of this optional panel automatically de-activates the "charge map approx" and "Dielectric and Kappa map approx" panels.

By default, the GUI is preconfigured to study biomolecules under normal physiological conditions. A monovalent $\mathrm{NaCl}$ salt solution at $0.15 \mathrm{M}$ concentration is predefined in the "ionic species (optional)" panel. Additionally, the solvent and solute dielectric coefficients are predefined to model water $\left(\varepsilon_{w}=78.54\right)$ and proteins $\left(\varepsilon_{p}=2\right)$ at room temperature $(\mathrm{T}=298.15 \mathrm{~K})$, respectively. These values can be changed as needed.

The "molecular structure" panel enables the user to upload molecular structures in either pdb or pqr format (see Figure 8). At least one file must be uploaded (molecule 1). For binding energy calculations, the user must also upload the molecular structures corresponding to the second biomolecule (molecule 2) and the complex system (molecule 1 plus molecule 2). It is not mandatory to upload the reference system file when only one molecule is uploaded (molecule 1). The pqr format is preselected to upload molecular structures. If the user does not have pqr file(s), the user must provide the corresponding pdb file(s). In the case neither pqr nor pdb file(s) is available, the user may use the "Need pdb files?" button to get the molecular structures from the protein data bank website (internet access is required). MPBEC automatically converts pdb into pqr files using pdb2pqr and AMBER (http://ambermd.org/), one of the most popular force fields used in biomolecular electrostatic calculations. The force field is defined in the file " /MPBEC/program/src/ Main/pdb2pqrscript.st" and it can be changed at any time. The uploaded molecular structure of each of the molecules can be visualized by clicking on the corresponding visualization button (“visualize mol 1?”, “visualize mol 2?”, “visualize complex?”).

The "write out files" panel, located at the middle of the main panel, is preselected to write out the mean electrostatic potential solution file (in $\mathrm{dx}$ format) and the surface plots for postanalysis purposes. The user is also able to generate map files by clicking on the corresponding blank boxes. The "output directory" box must be used to select a pre-existing directory to save the output files. By default, the software preselects the output directory “ MPBEC/program/src/examples/solutions”. MPBEC creates one folder in the selected output directory to save data corresponding to the target (fine) grid calculation. If the user selects "Optional Focus Calculation", MPBEC creates a second folder to save data 
corresponding to the focus (coarse) calculation. Each of these folders may contain several files to know: the logout file, the input files, the maps files, the energy file, the mean electrostatic potential file, the propka file and the surface plots, depending on the required calculations and selected options.

The "grid information" panel is located below the "write out files" panel. In the preselected "automatic" option, the GUI estimates the box size and the number of grid points based on the dimensions of the biological system. The GUI generates a box size 1.5 times larger than the length of the system along each direction. For average size systems smaller than $100 \AA$, the "automatic" option uses a fine mesh size less than or equal to $0.5 \AA$ and number of grid points less than or equal to 300 along each direction. These conditions provide good mesh resolution while preventing excessive computational cost and massive use of RAM memory. Otherwise, the "automatic" option uses the focusing boundary condition. Alternatively, the user may run MPBEC using the "manual" option. In this case, the user must provide the information on the box size and number of grid points along each of the Cartesian axis. For large biomolecules, the "Optional focus calculation" is more efficient than the corresponding target (fine) grid calculations. Another advantage of using focus calculation is to ensure that the a priori imposed Dirichlet boundary condition is satisfied. Further discussion on this topic is provided in Appendix B.

The "Linear Solver Setup" panel enables the user to select the Matlab linear solver algorithm ("Biconjugate gradient", "Gmres", "Minres") and to define the error tolerance ("Digits of precision") required on the mean electrostatic potential solution. The Biconjugate gradient method and 6 digits of precision are preselected by default. The "Boundary Condition" panel has two options. While computationally more expensive, the preselected "Dirichlet $(m d h)$ " option usually provides more accurate boundary conditions than "Dirichlet (sdh)". More details on these boundary conditions can be found in Appendix B.

The "input file name" panel is used to provide the input file name which must end with the extension ".inm". MPBEC uses the input file name to create output file names.

The "Energy Calculations (optional)" panel is located in the right-hand lower quarter of the main panel. By default, there is no preselected energy calculation. The user may select one or more energetic calculations depending on the number of uploaded biomolecules. For one biomolecule (e.g. molecule 1), the user may select three options: "Electrost. Pot. Energy" (Electrostatic potential energy), "Solvation free Energy" and/or " $p K a$ ". In the later case, the user must provide the " $p H$ " level of the aqueous electrolyte solution. On the other hand, the "Binding Energy" calculation can only be selected in the case that the user uploads the files corresponding to two biomolecules (protein and ligand) and the complex system.

\section{Solver Parameters and Algorithm Options}

\subsection{Discretization of the physical parameters involved with the solution of the PB equation}

The accuracy of the mean electrostatic potential solution depends on the algorithms and parameters used in the discretization of the charge density distribution and the kappa and dielectric functions appearing into the PB Eq. (1). 
Charge density map (chm)-The position of the atoms obtained from the pdb or pqr file may not coincide with any of the grid points. The $\mathrm{chm}$ panel has the following options to specifies the algorithm used to spread the point charges (summation term in Eq. 1) on the grid points.

SPLO, Trilinear interpolation (linear splines). This method locates the charge of each atom in the nearest neighbor grid point. The resulting charge density map may not be a smooth function, having sharp corners at the data points. SPLO has been shown to be an efficient algorithm to generate charge density maps but the accuracy of the PB solution may depend on the mesh, the box size, and the protein structure.

SPL2, Cubic B-spline interpolation (basis spline). This option results in the location of the charge in the nearest and next-nearest grid points. Whereas this calculation requires higher computational cost than SPLO, it provides softer distributions of charges which in turn generate mean electrostatic potential solutions less sensitive to the "Grid information" parameters. More information on interpolating splines can be found in reference [5].

Dielectric and kappa maps (dkm)-This panel specifies the algorithm and parameters used to build up the dielectric (first term in Eq. 1) and the kappa maps (second term in Eq. $1)$.

MOL: The dielectric coefficients are assigned according to the definition of the solvent accessible surface. This surface is calculated using the Collony molecular surface approach [6]. A solvent sphere is used to probe the molecule shape and create a surface defined by the union of the solvent-sized spheres, equidistant to the surface atoms in the molecule. The radius of the solvent probe "srad" is preassigned with the value $1.4 \AA$, which represents the radius of a water molecule (see Figure 1). The "surf dens" parameter is usually set equal to 10 and represents the number of points per $\AA^{2}$ used in the calculation of the Collony molecular surface. The volume outside the solvent accessible surface is assigned with "solvent dielectric coefficient" (preassigned as 78.54) whereas the rest of the space is assigned with the "solute dielectric coefficient" (preassigned as 2). Setting "srad" equal to zero generates a typical van der Waals surface. The ion-accessibility map (kappa map) is built up on a modified van der Waals surface. The radius of each atom in the molecule is inflated with the radius of the ion species (See "Ionic species" panel in Figure 1). The space inside the modified surface is assigned with an ion-accessibility coefficient equal to zero whereas the volume outside of this surface is assigned with the corresponding kappa values. The MOL method is very efficient, mainly for large proteins. However, the abrupt transition of the dielectric and kappa maps at the solute-solvent interface may give rise to discontinuous variations of the electrostatic free energies.

SMOL: This option includes a harmonic interpolation that smooths the surface on the dielectric and kappa maps generated by MOL, which reduces the sensitivity of the PB solution to "Grid information" parameters at the expense of increasing the computational cost [7]. 
SPL2: More suitable approaches (compared to MOL and SMOL) are required in those calculations demanding the evaluation of the slope of the mean electrostatic potential (e.g., electric force). In this case, the dielectric and kappa maps are built up on a cubic-spline surface where an intermediate dielectric region is introduced at the solute-solvent boundary to smooth the transition. The parameter "Swin" specifies the rate of change (e.g. the window width) in the dielectric interface [8]. The preassigned value for this parameter is $0.3 \AA$.

\subsection{Energy calculations}

MPBEC incorporates several free energy calculations which are of significant importance in biophysics and biomedicine. The free energy calculations are usually divided into two contributions: a nonpolar and a polar contribution. MPBEC calculates only the polar contribution to the free energy. Although the polar free energy calculations alone are not always sufficient to explain biological phenomena, they are the foundation for more complex, electrostatic calculations. A detailed description of polar free energy calculations can be found in reference [9].

The "Energy Calculations (optional)" panel provides the user four free energy calculations $(\mathrm{kJ} / \mathrm{mol})$ which may be chosen depending on the number of uploaded molecular structures.

Electros. Pot. Energy-MPBEC calculates the total electrostatic potential free energy stored in a single molecule. This energy represents the work required to assemble the molecule. It is calculated as

$$
G_{\text {elec }}=\frac{1}{2} \sum q_{i} \Phi\left(\mathbf{r}_{\mathbf{i}}\right)
$$

where $\Phi\left(\mathbf{r}_{\mathbf{i}}\right)$ is the mean electrostatic potential acting on the atom $i$ located at the position $\mathbf{r}_{\mathbf{i}}$ and carrying on charge $q_{i}$.

Solvation free energy-MPBEC calculates the polar contribution to the free energy released during the solvation of the biomolecule from vacuum to the solvent of interest as follows

$$
\Delta G=G_{\text {elec }}^{\text {Solv }}-G_{\text {elec }}^{R e f}
$$

where $G_{\text {elec }}^{\text {Ref }}$ (reference energy) is the total electrostatic potential free energy of the molecule in the reference state (solute uniform dielectric medium), whereas $G_{\text {elec }}^{\text {Solv }}$ (solvated energy) is that one in the solvated state (inhomogeneous dielectric medium formed by a cavity of solute dielectric constant surrounded by a solvent dielectric constant). In this calculation, the values of the solute and solvent dielectric constants are preassigned as 2 and 78.54, respectively.

Binding energy-MPBEC calculates the polar contribution to the binding free energy between two molecules (protein and ligand) defined by 


$$
\Delta G_{\text {binding }}=G_{\text {elec }}^{\text {complex }}-G_{\text {elec }}^{\text {protein }}-G_{\text {elec }}^{\text {ligand }}
$$

Although this approach does not account for conformational changes of the complex after formation, it is a good approximation to study interactions between biomolecules.

$\mathbf{p K a}$-Protein active sites are able to protonate/deprotonate residues depending on the number of hydrogen ions ( $\mathrm{pH}$ level) present in the aqueous electrolyte solution. This titration process may affect the function, stability, and molecular recognition of proteins. MPBEC uses PROPKA (www.propka.org) to calculate the protonation (positioning of hydrogen ions) to a given molecular structure (in pdb format) at the specified $\mathrm{pH}$ level [10]. PROPKA generates a new pdb file containing the re-normalized protein charge distribution, which is subsequently used to solve the linear PB equation.

\subsection{Focus calculations}

In focusing calculations, the Matlab script solves the PB equation twice automatically. The first time it calculates the solution in the coarse (large) grid box and, subsequently, it uses this solution on the faces of the fine (small) grid box as the boundary condition to calculate the solution in the fine grid. Note that the Coarse box size must be greater than the fine box size. More information on focusing calculation is provided in Appendix B.

\subsection{Linear equation system solver}

The Linear Solver Setup panel provides three options ("Biconjgrad", "Gmres", and "Minres") to select the algorithm that MPBEC uses to numerically solve $A u=b$, namely the discretized version of the linear PB Eq. 1. The square matrix $A$ contains the information on the dielectric and kappa maps, the column vector $b$ contains the information on the charge map and boundary condition, and $u$ represents the unknown discretized dimensionless mean electrostatic potential vector. More details on the theoretical approach is given in Appendix B.

MPBEC uses sparse matrix calculations and iterative linear solvers to solve the discretized linear PB equation. The stability and the convergence of these iterative solvers is improved by using the incomplete LU factorization Matlab function $\{L, U\}=i l u(A, d r o p t o l)$, which generates pre-conditioner matrices $L$ (lower triangular matrix) and $U$ (upper triangular matrix) at low computational cost. The tolerance droptol in calculating the incomplete LU factorization is set equal to 0.000005 . The three solvers require the input of the initial guess value $u o$, the solver maximum iteration number maxit, and the error tolerance tol. MPBEC uses the following values for these parameters: $u o=0$, maxit $=400$, and $t o l=10^{-} p$, where $p$ is the "digits of precision" required by the user.

Gmres-MPBEC calls the Matlab function [u,flag]=gmres(A,b,restart,tol,maxit,L,U,uo) to solve the linear equations system using generalized minimal residual method. The solver is automatically restarted after 10 iterations. 
Minres-MPBEC calls the Matlab function $[u, f l a g]=\operatorname{minres}(A, b$, tol, maxit $L, U, u o)$ to solve the linear equations system using minimal residual method.

Biconjgrad-MPBEC calls the Matlab function bicgstab $(A, b, u$, tol,maxit, $L, U, u o)$ to solve the linear equations system using biconjugate gradients stabilized method.

We notice that none of the aforementioned three options is the best for all biomolecules and aqueous electrolyte solutions (e.g. for all matrices $A$ and vectors $b$ ). There are always examples in which one option outperforms the other. Therefore, multiple solvers should be tried in practice to see which one is the best for a given problem. The generalized minimal residual method ("Gmres") is an extension of the minimal residual method ("Minres") which is only applicable to symmetric systems. Like the minimal residual method, "Gmres" generates a sequence of orthogonal vectors, but in the absence of symmetry this can no longer be done with short recurrences; instead, all previously computed vectors in the orthogonal sequence have to be retained at the cost of a larger storage demand. For this reason, "restarted" versions of the method are used to avoid excessive storage requirements and computational cost for the orthogonalization. On the other hand, the "Minres" method is very vulnerable for rounding errors. The biconjugate gradient method takes another approach, replacing the orthogonal sequence of residuals by two mutually orthogonal sequences, at the price of no longer providing a minimization. Hence, the residual does not decrease monotonically, and consequently the convergence is not guaranteed. Based on our experience, the "Biconjgrad" option performs well for all the proteins provided in the example section. If this method does not converge, MPBEC automatically uses "Gmres", and subsequently "Minres", if needed. More information on iterative methods for solving linear equations can be found in reference [11].

\section{Computer requirements}

Java and Matlab must be installed on the computer. MPBEC was successfully tested on PC Windows 7 and Windows 8.1, Linux Fedora 16 and Fedora 19, and OSX Yosemite running Matlab R2010a and newer versions. We recommend to run MPBEC on computers having at least $2 \mathrm{~GB}$ of free RAM memory and $2.5 \mathrm{GHz}$ CPU speed or higher. Some examples of estimated RAM memory allocations required by the automatic grid configuration to run MPBEC are provided in Table 7. As it happens with other applications, it is recommended to keep only one copy of the MPBEC software in your computer to avoid potential Matlab path search issues.

\section{Examples}

In this section we present the results obtained for several biological systems to illustrate the use of different solver algorithms and parameters as well as the solver performance. We also compare MPBEC against APBS (version 1.4) results for testing purposes. In all these systems we use the following parameter values: solvent dielectric coefficient $=78.54$, temperature $=298.15 \mathrm{~K}$, boundary condition in the fine grid $=\mathrm{mdh}$, solver method $=$ biconjgrad, and digits of precision= 6 . We also use the "manual" option to setup the grid 
information unless otherwise is stated. The molecular structure input files used in this section can be found in the directory $\sim / \mathrm{MPBEC} /$ program/src/examples.

Table 1 shows the polar solvation free energy results obtained for the Born ion in a box of $32 \AA \times 32 \AA \times 32 \AA$ for different number of grid points and, consequently, different grid spacing. We use the "Solvation free energy" option in the "Energy calculations" panel, and set the ionic concentrations equal to $0, \mathrm{chm}=\mathrm{SPL} 0, \mathrm{dkm}=\mathrm{MOL}$, and solute dielectric coefficient= 1. Table 2 shows the polar solvation free energy results obtained for the same biological system using 193 grid points along each direction, and different charge (SPL0, SPL2) and dielectric (MOL, SMOL, SPL2) approximations. Tables 3 and 4 show the polar solvation free energy results obtained for Methanol and Methoxide (ionized methanol), respectively, in a box of $32 \AA \times 32 \AA \times 32 \AA$ for different charge approximations (SPLO, SLP2). These molecules are in the presence of a single monovalent salt $(\mathrm{NaCl})$ with ionic radius $=2 \AA$ and $0 \mathrm{M}, 0.05 \mathrm{M}$, and $0.5 \mathrm{M}$ salt concentrations. We use 193 grid points along each direction and set $\mathrm{dkm}=\mathrm{MOL}$, and solute dielectric coefficient $=2$. In these examples we observe that the solvated and reference state energies are sensitive to the different parameters and approximations whereas the polar solvation free energy remains approximately constant.

Tables 5 and 6 illustrate the use of the "Binding energy" and "Optional Focus calculation" options for two complex systems, namely hca (protein)-acetazolamide (ligand) and $1 \mathrm{~d} 7 \mathrm{~h}$ (protein)-DMSO (ligand). We use the "Optional Focus calculation" with 129 grid points along each direction, a focus (coarse) box of $96 \AA \times 96 \AA \times 96 \AA$ and a target (fine) box of $57 \AA ̊ \times 57 \AA ̊ 57 \AA$. We also set $\mathrm{dkm}=\mathrm{MOL}, \mathrm{chm}=\mathrm{SPL} 0$, and solute dielectric coefficient $=2$. While the hca-acetazolamide complex is not immersed in a electrolyte, the $1 \mathrm{~d} 7 \mathrm{~h}$-DMSO complex is in presence of a monovalent salt with concentration of $0.01 \mathrm{M}$ and ionic radius of $2 \AA$.

Figure 9 illustrates the use of the " $p K a$ " option. It presents the surface potential of the protein $2 \mathrm{KAC}$ at three $\mathrm{pH}$ levels (12, 7, and 3). In this case, we select the "Automatic" option in the "grid information" panel and use the preassigned parameters. The propka file saved in the "output directory" provides useful information about the charge renormalization at several $\mathrm{pH}$ levels. In particular, the total protein charge increases from $-15 \mathrm{e}$ to $0.7 \mathrm{e}$ when the $\mathrm{pH}$ level decreases from 7 to 3 , whereas it decreases from $-15 \mathrm{e}$ to $-20 \mathrm{e}$ when the $\mathrm{pH}$ level increases from 7 to 12 . Additionally, the isoelectric point predicted for this protein is 3.33 .

Table 7 presents the polar solvation free energy results obtained for several proteins using the preselected parameters and the "automatic" grid information option. We also include the registered computing time obtained for proteins of different sizes using Matlab R2012b in a single CPU Intel Xeon X5670 Westmere 2.93 GHz under Linux Fedora 19.

\section{Supplementary Material}

Refer to Web version on PubMed Central for supplementary material. 


\section{Acknowledgments}

Marcelo Marucho would like to acknowledge support from NIH Grant No. 1SC2GM112578-01. The authors would like to thank Dr. Ariel Balter from Pacific Northwest National Laboratory for his useful comments and suggestions on the validation of MPBEC. The authors also thank Dr. Nathan A. Baker from Pacific Northwest National Laboratory for his insightful comments on the development of MPBEC. He would like to acknowledge support from NIH Grant No. 5R01GM069702.

\section{AppendixA. How to Run MPBEC without Using the GUI}

Running MPBEC without using the GUI is not recommended unless MPBEC is implemented into other Matlab programs or the user has Matlab programming and text editing skills as well as strong background in biophysics. The user must first include the Matlab search path corresponding to the folder /MPBEC and subfoders from the Matlab console. It must also generate the input file named "inputfile.inm" using any text editor (see input file structure section for instructions). This file must be saved in the directory / MPBEC/program/src/Main. Then, the user has to verify that the current folder in Matlab search toolbar is /MPBEC/program/src/Main before running MPBEC. The user also must check that the biological system satisfies the electroneutrality condition, unusual/ nonphysical values are not assigned to the required parameters, or any input data is missing, among other pre-tests the GUI does automatically. Finally, the user has to type MPBEC('inputfile.inm') on the Matlab commander window and press Enter to run the MPBEC one argument MATLAB function. The implementation of MPBEC into existing Matlab programs is straightforward since MPBEC is a single argument Matlab function. Additionally, Matlab parallel computing toolbox may be used to run MPBEC in multiple processors.

\section{AppendixA.1. Input File structure (fine grid calculations)}

The "inputfile.inm" file parsing is strict. Input data must contain the value of these parameters in the exact following column order (see example in Figure AppendixA.1):

$$
\begin{aligned}
& \text { dime } \\
& \text { glen } \\
& \text { bulk } \\
& \text { bc } \\
& \text { solversetup } \\
& \text { dielx_str } \\
& \text { diely_str } \\
& \text { dielz_str } \\
& \text { kappa_str } \\
& \text { charge_str } \\
& \text { pqr_str }
\end{aligned}
$$




$$
\begin{aligned}
& \text { pqr_cent_str } \\
& \text { energy } \\
& \text { in_name_str } \\
& \text { name_str } \\
& \text { wout }
\end{aligned}
$$

\section{AppendixA.1.1. Description of each arrow}

dime This line is used to specify the number of grid points. The values for this keyword are: $\boldsymbol{n} \boldsymbol{x} \boldsymbol{n y} \boldsymbol{n z}$ which represent the (integer) number of grid points in the $\mathrm{x}^{-}, \mathrm{y}-$, and $\mathrm{z}$-directions, respectively.

glen This line is used to specify the mesh domain lengths. The values for this keyword are: xlen ylen zlen which represent (floating point) grid lengths $L_{x}, L_{y}$ and $L_{z}$ (in $\AA$ ) along the x-, $\mathrm{y}$-, and z-directions, respectively.

bulk This line is used to specify bulk properties and parameters involved in the calculation of the dielectric and kappa maps. The values for this keyword are: Ionicstrength maxionR dielw dielp T srad surfdens swin. Ionicstrength defines the value of the ionic strength $I=\frac{1}{2} \sum c_{i} z_{i}^{2}$ where he sum is over all different ionic species " $i$ ", and $z_{i}$ and $c_{i}$ are the ionic valence and concentration $\mathrm{P}$ (in moles), respectively. This quantity is used in the calculation of the Dirichlet boundary condition and the kappa map. maxion $\mathbf{R}$ is the maximum ionic radius (in $\AA$ ) used in MOL and SMOL approaches to calculate the kappa map. dielw is the value of the solvent dielectric coefficient involved in the calculation of the dielectric map. It is usually set equal to 78.54 for water in the solvated state and equal to the solute dielectric coefficient in the reference state. dielp is a number, usually between 2 and 10, which defines the value of the solute dielectric coefficient involved in the calculation of the dielectric map. $\mathbf{T}$ is the temperature of the system in Kelvin degrees. srad defines the molecular solvent radius in $\AA$ used in MOL and SMOL approaches to calculate the Collony molecular surface. It is usually set equal to 1.4 to model water. surfdens is usually set equal to 10 and represents the number of points per $\AA^{2}$ used in MOL and SMOL approaches to calculate the Collony molecular surface. swin defines the value of the window width parameter (in $\AA$ ) used in SPL2 approach to calculate the dielectric and kappa maps, usually set equal to 0.3.

bcThis line is used to specify the boundary condition used in the calculation of the mean electrostatic potential. The words for this keyword are either mdh (if the user requires multiple Dirichlet boundary condition), sdh (if the user requires single Dirichlet boundary condition), or focusname.inm (if the user requires focus boundary condition).

solversetup This line is used to specify the linear solver setup. The words for this keyword are: digpres selectsolver. digpres is the number of digits of precision required on the solution of the linearized PB equation. This number is usually set equal to 6 . selectsolver specifies the linear solver method. The words for this keyword are either biconjgrad (it uses Biconjugate Gradient solver stabilized by LU decomposition), gmres (it uses Generalized 
minimal residual solver stabilized by LU decomposition), or minres (it uses the minimal residual solver).

dielx_str This line is used to specify the file name of the shifted x-component of the dielectric coefficients in $\mathrm{dx}$ format (for instance dielx.dx). If this file is not uploaded, the user must set this keyword equal to nouploadedfiles.

diely_strSame description provided for dielx_str but in this case the file name refers to the shifted y-component of the dielectric.

dielz_str Same description provided for dielx_str but in this case the file name refers to the shifted z-component of the dielectric.

kappa_str This line is used to specify the file name of the ionic accessibility coefficients kappa in dx format (for instance kappa.dx). If this file is not uploaded, the user must set this keyword equal to nouploadedfiles.

charge_str This line is used to specify the file name in dx format of the charge density coefficients in units of $1 / \AA^{3}$ (for instance charge.dx). If this file is not uploaded, the user must set this keyword equal to nouploadedfiles.

pqr_str This line is used to specify the pqr file name of the molecular structure (for instance moleculetarget.pqr). This file determines the target molecule to solve the PB equation.

pqr_cent_str This line is used to specify the pqr file name of the molecule which contains the molecular structure that defines the center of grid. If there is only one molecule in the system, pqr_cent_str and pqr_str must contain the same keyword. In systems having two molecules pqr_cent_str specifies the pqr file name corresponding to the reference molecule. In such case the center of grid is defined by the coordinates of that molecule for all the molecules comprised by the complex system. An example of this file name is moleculereference.pqr.

$\mathbf{d k m}$ This line is used to specify the method used in the calculation of the dielectric and kappa maps. The words for this keyword are either MOL (if the user requires the Collony molecular surface approach), SMOL (if the user requires the harmonic smoothing approach of the Collony molecular surface), or SPL2 (if the user requires cubic spline approach).

chm This line is used to specify the method in the calculation of the charge density map. The words for this keyword are either SPLO (if the user requires linear interpolating approach) or SPL2 (if the user requires cubic interpolating approach).

energy This line is used to specify the calculation of the total electrostatic potential free energy given by Eq.(2). The words for this keyword are either calceneryes (if the user requires energy calculations), or calcenerno (if the user does not require energy calculations).

in_name_strThis line is used to specify the full path to the input file directory containing all the files to be uploaded. An example of the input file directory is 
C:Ismith\Documents\MPBEClprogramlsrclexamples\pqr

for Windows users and

/home/smith/Documents/MPBEC/program/src/examples/pqr

for Linux and Mac users.

name_str This line is used to specify the full path to the output file directory that contains the resulting output files. For instance, this directory may be

IMPBEClprogram\srclexamples\solutions

for Windows users and

/MPBEC/program/src/examples/solutions

for Linux and Mac users.

wout This line is used to specify the required output files. The values for this keyword are: n1 n2 n3 n4 n5. n1 set equal to 1.0 writes out the charge map (dx file) whereas n1 set equal to 0.0 does not. Similarly, $\mathbf{n} \mathbf{2}, \mathbf{n 3}, \mathbf{n} \mathbf{4}$, and $\mathbf{n 5}$ are those parameters corresponding to the kappa map ( $\mathrm{dx}$ file), dielectric maps (dx files), mean electrostatic potential solution ( $\mathrm{dx}$ file), and electric potential surface plots, respectively.

\section{AppendixA.2. Input File structure (coarse grid calculations using focusing)}

For focusing calculations, the user has to generate two input files. One of them, the "inputfile.inm" file, specifies the parameters required to perform the calculation on the fine grid. Note that the keyword focusname.inm must be assigned in the bc line. The second input file, named "focusname.inm", provides the parameters corresponding to the focusing calculation using the same file structure described in the previous section for the "inputfile.inm" file. The files "inputfile.inm" and "focusname.inm" must be saved in the directory /MPBEC/program/src/Main. To run MP-BEC on the Matlab commander window, the user has to type MPBEC('inputfile.inm') and press enter. 


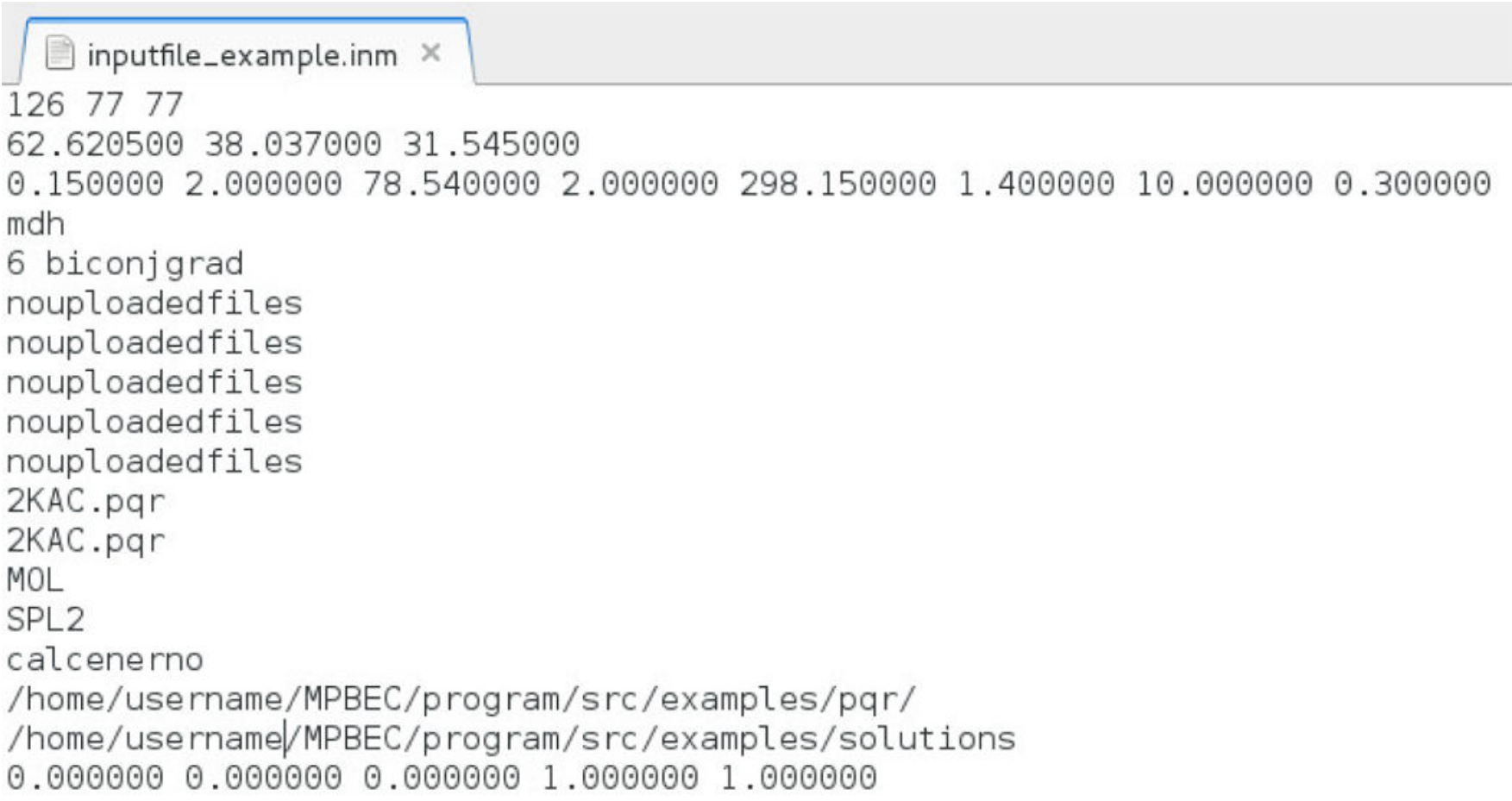

Figure AppendixA.1.

Input file example to run MPBEC without using the GUI.

\section{AppendixB. Computational Approach}

\section{AppendixB.1. Theoretical formulation}

MPBEC calculates the numerical solution of the linearized PB equation (1) using the boxmethod proposed by Michael Holst [12]. For a diagonal dielectric tensor, the resulting discretized linear PB equations at the nodes $u_{i j k}=u\left(x_{i}, y_{j}, z_{k}\right)$ for $1 \leq i \leq N_{x}, 1 \leq j \leq N_{y}$ and 1 $\leq k \leq N_{z}$ reads

$$
\begin{gathered}
{\left[\epsilon_{i-1 / 2, j, k}^{x} \frac{\left(h_{j-1}+h_{j}\right)\left(h_{k-1}+h_{k}\right)}{4 h_{i-1}}+\epsilon_{i+1 / 2, j, k}^{x} \frac{\left(h_{j-1}+h_{j}\right)\left(h_{k-1}+h_{k}\right)}{4 h_{j}}+\right.} \\
\epsilon_{i, j-1 / 2, k}^{y} \frac{\left(h_{i-1}+h_{i}\right)\left(h_{k-1}+h_{k}\right)}{4 h_{j}-1}+\epsilon_{i, j+1 / 2, k}^{y} \frac{\left(h_{i-1}+h_{i}\right)\left(h_{k-1}+h_{k}\right)}{4 h_{j}}+ \\
\epsilon_{i, j, k-1 / 2}^{k} \frac{\left(h_{i-1}+h_{i}\right)\left(h_{j-1}+h_{j}\right)}{4 h_{k-1}}+\epsilon_{i, j, k+1 / 2}^{k} \frac{\left(h_{i-1}+h_{i}\right)\left(h_{j-1}+h_{j}\right)}{4 h_{k}}+ \\
\left.\kappa_{i j k} \frac{\left(h_{i-1}+h_{i}\right)\left(h_{j-1}+h_{j}\right)\left(h_{k-1}+h_{k}\right)}{8}\right] u_{i j k}+ \\
{\left[-\epsilon_{i-1 / 2, j, k}^{x} \frac{\left(h_{j-1}+h_{j}\right)\left(h_{k-1}+h_{k}\right)}{4 h_{i-1}}\right] u_{i-1 j k}+\left[-\epsilon_{i+1 / 2, j, k}^{x} \frac{\left(h_{j-1}+h_{j}\right)\left(h_{k-1}+h_{k}\right)}{4 h_{i}}\right] u_{i+1 j k}+} \\
\left.\left[-\epsilon_{i, j-1 / 2, k}^{y} \frac{\left(h_{i-1}+h_{i}\right)\left(h_{k-1}+h_{k}\right)}{4 h_{j-1}}\right] u_{i j-1 k}+-\epsilon_{i, j+1 / 2, k}^{y} \frac{\left(h_{i-1}+h_{i}\right)\left(h_{k-1}+h_{k}\right)}{4 h_{j}}\right] u_{i j+1 k}+ \\
{\left[-\epsilon_{i, j, k-1 / 2}^{k} \frac{\left(h_{i-1}+h_{i}\right)\left(h_{j-1}+h_{j}\right)}{4 h_{k-1}}\right] u_{i j k-1}+\left[-\epsilon_{i, j, k+1 / 2}^{y} \frac{\left(h_{i-1}+h_{i}\right)\left(h_{j-1}+h_{j}\right)}{4 h_{j}}\right] u_{i j k+1}=} \\
\frac{4 \pi e_{c}^{2}\left(h_{i-1}+h_{i}\right)\left(h_{j-1}+h_{j}\right)\left(h_{k-1}+h_{k}\right)}{K_{B} T} f_{i j k}
\end{gathered}
$$

where the grid mesh is defined by

$$
h_{i}=x_{i+1}-x_{i}, h_{j}=y_{j+1}-y_{j} h_{k}=z_{k+1}-z_{k}
$$


The Dirac delta functions appearing in the right hand side of the starting equations are approximated with spline functions which spread the point like charge positions along the neighborhood grid points. The resulting coefficients fijk represent the smearing of the point charges along the grid points. For example, the explicit expressions for the equations defined in a grid of $5 \times 5 \times 5$ number of points are

$$
\begin{gathered}
a_{222} u_{222}+a_{122} u_{122}+a_{322} u_{322}+a_{212} u_{212}+a_{232} u_{232}+a_{221} u_{221}+a_{223} u_{223}=f_{222} \\
a_{322} u_{322}+a_{222} u_{222}+a_{422} u_{422}+a_{312} u_{312}+a_{332} u_{332}+a_{321} u_{321}+a_{323} u_{323}=f_{322} \\
a_{422} u_{422}+a_{122} u_{122}+a_{322} u_{322}+a_{212} u_{212}+a_{232} u_{232}+a_{221} u_{221}+a_{223} u_{223}=f_{422} \\
a_{232} u_{232}+a_{132} u_{132}+a_{332} u_{332}+a_{222} u_{222}+a_{242} u_{242}+a_{231} u_{231}+a_{233} u_{233}=f_{232} \\
\ldots
\end{gathered}
$$

The nodes can be arranged using the natural ordering

$$
u=\left[u_{111}, u_{211}, . ., u_{N_{x} 11}, u_{121}, . ., u_{221}, u_{321}, . ., u_{N_{x} 21} \ldots, u_{N_{x} N_{y} N_{z}}\right]^{T}
$$

The prescribed values of nodes $u_{1 j k}, u_{N_{x}, j, k} u_{i, 1, k}, u_{i, N_{y}, k}, u_{i j 1}$ and $u_{i j N_{z}}$ along the faces of the box coming from the Dirichlet boundary conditions are conveniently brought to the righthand side of the equations. The resulting left-hand side equations only contain the following interior (unknown) nodes

$$
u=\left[u_{222}, u_{322}, . ., u_{N_{x}-1,22}, u_{232}, . ., u_{332}, u_{432}, . ., u_{N_{x}-2,32 \ldots}, u_{N_{x}-1, N_{y}-1, N_{z}-1}\right]^{T}
$$

in such a way that the previous equations become

$$
\begin{gathered}
a_{222} u_{222}+a_{322} u_{322}+a_{232} u_{232}+a_{223} u_{223}=f_{222}-a_{122} u_{122}-a_{212} u_{212}-a_{221} u_{221} \equiv b_{222} \\
a_{322} u_{322}+a_{222} u_{222}+a_{422} u_{422}+a_{332} u_{332}+a_{323} u_{323}=f_{322}-a_{312} u_{312}-a_{321} u_{321} \equiv b_{322} \\
a_{422} u_{422}+a_{322} u_{322}+a_{232} u_{232}+a_{223} u_{223}=f_{422}-a_{122} u_{122}-a_{212} u_{212}-a_{221} u_{221} \equiv b_{422} \\
a_{232} u_{232}+a_{332} u_{332}+a_{222} u_{222}+a_{242} u_{242}+a_{233} u_{233}=f_{232}-a_{132} u_{132}-a_{231} u_{231} \equiv b_{232}
\end{gathered}
$$

These set of equations can be written in compact form in term of matrix vector product as follows

$$
A u=b \quad \text { (B.2) }
$$

in which

$$
\begin{gathered}
u(p)=u_{i j k}, \quad b(p)=b_{i j k}, \quad p=(k-2)\left(N_{x}-2\right)\left(N_{y}-2\right)+(j-2)\left(N_{x}-2\right)+i-1 \\
i=2, . ., N_{x}-2, \quad j=2, . ., N_{y}-2, \quad k=2, . ., N_{z}-2
\end{gathered}
$$

and $A$ is a $\left(N_{x}-2\right)\left(N_{y}-2\right)\left(N_{z}-2\right)$ by $\left(N_{x}-2\right)\left(N_{y}-2\right)\left(N_{z}-2\right)$ seven banded block squared symmetric matrix which contains the following nonzero elements:

- The main diagonal elements 


$$
\begin{gathered}
d_{0}(p)=\left[\epsilon_{i-1 / 2, j, k}^{x} \frac{\left(h_{j-1}+h_{j}\right)\left(h_{k-1}+h_{k}\right)}{4 h_{i-1}}+\epsilon_{i+1 / 2, j, k}^{x} \frac{\left(h_{j-1}+h_{j}\right)\left(h_{k-1}+h_{k}\right)}{4 h_{i}}+\right. \\
\epsilon_{i, j-1 / 2, k}^{y} \frac{\left(h_{i-1}+h_{i}\right)\left(h_{k-1}+h_{k}\right)}{4 h_{j-1}}+\epsilon_{i, j+1 / 2, k}^{y} \frac{\left(h_{i-1}+h_{i}\right)\left(h_{k-1}+h_{k}\right)}{4 h_{j}}+ \\
\left.\epsilon_{i, j, k-1 / 2}^{k} \frac{\frac{\left(h_{i-1}+h_{i}\right)\left(h_{j-1}+h_{j}\right)}{4 h_{k-1}}+\epsilon_{i, j, k+1 / 2}^{k} \frac{\left(h_{i-1}+h_{i}\right)\left(h_{j-1}+h_{j}\right)}{4 h_{k}}+}{8}\right] \\
\left.\kappa_{i, j, k} \frac{\left(h_{i-1}+h_{i}\right)\left(h_{j-1}+h_{j}\right)\left(h_{k-1}+h_{k}\right)}{8}\right]
\end{gathered}
$$

- The next upper band diagonal, which is shifted in one column to the left from the first column, contains the following elements

$$
\left[d_{1}(p)=-\epsilon_{i+1 / 2, j, k}^{x} \frac{\left(h_{j-1}+h_{j}\right)\left(h_{k-1}+h_{k}\right)}{4 h_{i}}\right]
$$

- The second upper band diagonal which is shifted $N_{x}-2$ columns from the first column

$$
d_{2}(p)=\left[-\epsilon_{i, j+1 / 2, k}^{y} \frac{\left(h_{i-1}+h_{i}\right)\left(h_{k-1}+h_{k}\right)}{4 h_{j}}\right]
$$

- $\quad$ The third upper band diagonal which is shifted $\left(N_{x}-2\right)\left(N_{y}-2\right)$ columns from the first column

$$
d_{3}(p)=\left[-\epsilon_{i, j, k+1 / 2}^{k} \frac{\left(h_{i-1}+h_{i}\right)\left(h_{j-1}+h_{j}\right)}{4 h_{k}}\right]
$$

The remaining elements of the upper triangular squared matrix A are set equal to zero. By symmetry we obtain the lower tri-diagonal elements of the matrix A. Because the matrix A is sparse and large, we can implement efficient methods that optimally solve the linear system for $u$ as described below.

\section{AppendixB.2. Computational Scheme}

The computational scheme to be implemented by the user greatly depends on the available computational resources. MPBEC implements two computational schemes depending basically on the computation cost and computer memory required to solve the PB equation. The automatic computational algorithm without focusing calculation might be the right choice to obtain the numerical solution of the corresponding PB equation for small to medium molecule sizes, grid resolutions up to $0.5 \AA$ along each direction, and total number of points up to $300 \times 300 \times 300$ (like most of systems described in the example section of this article). On the other hand, large biomolecules, larger box sizes, and higher grid resolutions demand much higher computation cost and computer memory, making the computational algorithm with focusing calculation a better choice to perform the calculations. 


\section{AppendixB.2.1. Computational algorithm without focusing calculation}

1. Reading the input file. The code reads the input file to get the physical parameters (solvent and solute dielectric coefficients, charge distribution(s) and molecular structure(s), temperature, ion densities, charge valence and sizes) as well as the solver parameters (number of grid points, the box lengths, tolerance, method).

2. Calculation of the origin of the reference system and the mesh size. The center of the grid is calculated from the corresponding molecular structure(s) file(s). The values of the mesh size $h_{i}, h_{j}$ and $h_{k}$ are calculated from the number of grid points and the box size.

3. Generation of the maps and boundary conditions. The charge density is discretized using spline functions (SPL0 and SPL2 options) to get the coefficients $f_{i j k}$. The solvent and solute dielectric coefficients and the ionic strength are used to calculate the shifted dielectric coefficients $\epsilon_{i, j, k}^{x}, \epsilon_{i, j, k}^{y} \epsilon_{i, j, k}^{z}$ and kappa function $k_{i j k}$ elements, respectively, inside and outside the solvent-accessible molecular surface using the Collony surface approach (MOL, SMOL, and SPL2 options). The values of the nodes $u_{1 j k}, u_{N_{x}, j, k} u_{i, 1, k}, u_{i, N_{y}, k}, u_{i j 1}$ and $u_{i j N_{z}}$ along the six faces of the box, including the edges and the corner nodes $\left(1 \leq i \leq N_{x}, 1 \leq j \leq N_{y}\right.$ and $\left.1 \leq k \leq N_{z}\right)$, are set equal to the values prescribed by a Debye-Hückel model [13] (see Figure AppendixB.1) using the temperature, the value of the solvent dielectric coefficient (usually water), and the ionic strength. The "sdh" boundary condition option corresponds to the use of the mean electrostatic potential generated by a single solute sphere, with radius equal to the average molecule size radius and sphere charge, dipole, and quadrupole equal to the total moments of the molecule. This boundary condition provides better results when the box sides are sufficiently far from the biomolecule. In contrary, the "mhd" boundary condition option works better than "sdh" for box sides closer to the biomolecule. However, this condition can be very slow for large biomolecules since it uses the mean electrostatic potential generated by multiple, non-interacting spheres with a point charges, where the sphere radii are set to the atomic radii of the biomolecule and the sphere charges are set to the total charge of the molecule.

4. Generation of the seven banded block tri-diagonal matrix A. The expressions (B.3), (B.4),(B.5), (B.6), and the value of the shifted dielectric coefficients $\epsilon_{i, j, k}^{x}, \epsilon_{i, j, k}^{y}$, $\epsilon_{i, j, k}^{z}$ and kappa function $k_{i j k}$ are used to calculate the nonzero components of the matrix $A$, e.g., the diagonal elements $d_{0}(p), d_{1}(p), d_{2}(p)$, and $d_{3}(p)$, for $p=(k-2)$ $\left(N_{x}-2\right)\left(N_{y}-2\right)+(j-2)\left(N_{x}-2\right)+i-1$ and $i=2, . ., N_{x}-1, j=2, . ., N_{y}-1, k=$ $2, \ldots, N_{z}-1$. The sparse upper triangular matrix $\mathrm{A}$ is constructed by filling with zeros the remaining elements of the matrix A using the spdiag.m Matlab script. The lower triangular elements of the matrix $\mathrm{A}$ are obtained by using the symmetry property $A_{p q}=A_{q p}$ for $q=1, . .,\left(N_{x}-2\right)\left(N_{y}-2\right)\left(N_{z}-2\right)$ and $p=q, . .,\left(N_{x}-2\right)\left(N_{y}\right.$ $-2)\left(N_{z}-2\right)$.

5. Generation of the vector $b$. The discretized charge density $f_{i j k}$, the values of the Dirichlet boundary elements, and the shifted dielectric coefficient values are used 
to calculate the elements of $b_{i j k}$. The natural ordering defined in the previous step is used to construct the vector $b(p)$ from the corresponding data array structure $b_{i j k}$.

6. Solving PB equation inside the box. The inexact $L U$ decomposition of the matrix $A$ is performed by using the row-sum modified incomplete $L U$ factorization (ilu.m Matlab script) which provides a fast evaluation of the matrices $L$ and $U$. The resulting $L$ and $U$ matrices, the matrix $A$, and the vector $b$ are used to numerically solve the linear system of equations $A u=b$ for the vector $u$ using one of the following linear solvers: the biconjugate gradient stabilized method (bicgstab.m Matlab script), Gmres (gmres.m Matlab script), or Minres (minres.m Matlab script).

7. Building up the solution for the mean electrostatic potential. The natural ordering defined in step 4 is used to convert the resulting vector $u(p)$ into the data array structure $u_{i j k}$. The previously removed values of the nodes at the faces of the box are used to obtain the solution for the nodes $u_{i j k}$ over the complete set of grid points, namely for $i=1, \ldots, N_{x}, j=1, . ., N_{y}, k=1, \ldots, N_{z}$.

8. Energy calculations. The code uses the solution obtained for the mean electrostatic potential to perform required energy calculations.

9. Output files generation. The electrostatic potential $u_{i j}$, shifted dielectric coefficients $\epsilon_{i, j, k}^{x}, \epsilon_{i, j, k}^{y}, \epsilon_{i, j, k}^{z}$, kappa function $k_{i j k}$ elements, and the charge density $f_{i j k}$ maps are saved in $\mathrm{dx}$ format files in the folder assigned by the user if required.

An alternative algorithm is used when the user provides the dielectric and kappa function maps as input files. In this case, the algorithm uses these values to solve the PB equation instead of calculating them from the solvent and solute dielectric constants and ionic strength.

\section{AppendixB.2.2. Focusing Calculation}

Large biological systems require large box sizes in order to validate the implementation of the Dirichlet boundary condition in the calculation of the solution of the PB equation inside the box. In such case, the solution of the mean electrostatic potential at high resolution may demand high computation cost and computer memory. To overcome these limitations MPBEC implements the focusing calculation. This approach is based on the APBS focusing algorithm where the PB equation is solved in two steps. First, a large enough box size is used to obtain the solution of PB equation at low resolution and fast calculation using Dirichlet boundary condition. Secondly, a smaller box size containing the system is used to obtain the solution of PB equation at higher resolution. The solution obtained for the large box at lower resolution is used to calculate the boundary condition for the smaller box using linear interpolation. 


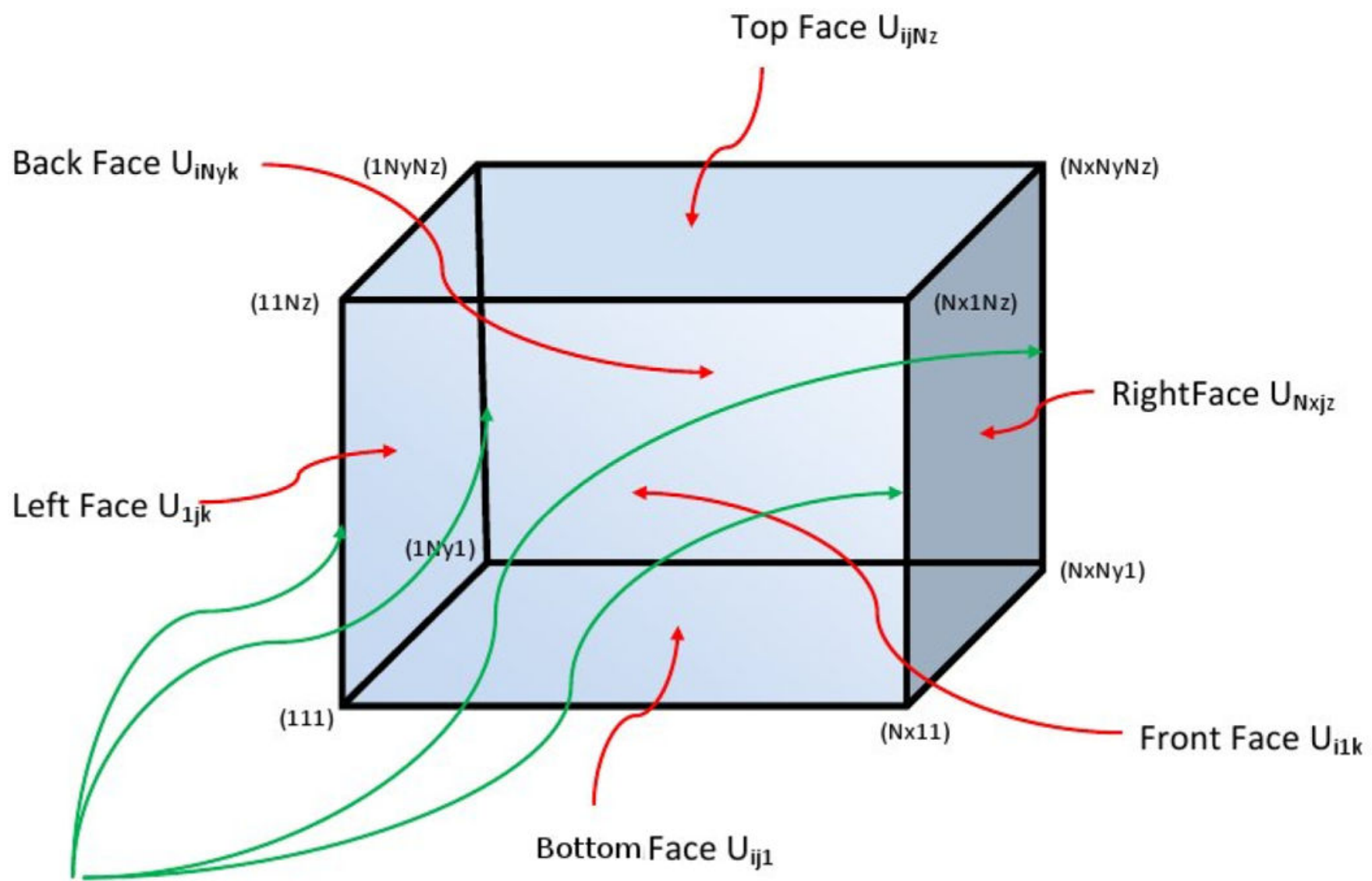

\section{Edges along z-direction}

Figure AppendixB.1.

Boundary condition

\section{References}

1. Ren P, Chun J, Thomas DG, Schnieders MJ, Marucho M, Zhang J, Baker NA. Biomolecular electrostatics and solvation: a computational perspective, Q. Rev Biophys. 2012; 45:427-491.

2. Rocchia, W.; Spagnuolo, M. Computational Electrostatics for Biological Applications. Springer; New York: 2015.

3. APBS (http://www.poissonboltzmann.org/); Delphi (http://compbio.clemson.edu/delphi); MEAD (http://www.scripps.edu/mb/bashford/); Zap (http://www.eyesopen.com/zap-tk); Charmm (http:// www.charmm.org/documentation/c34b1/pbeq.html).

4. Unni S, Huang Y, Hanson R, Tobias M, Krishnan S, Li WW, Nielsen JE, Baker NA. Web servers and services for electrostatics calculations with APBS and PDB2PQR. J Comput Chem. 2011; 32:1488-1491. [PubMed: 21425296]

5. Hämmerlin, G.; Hoffmann, KH. Numerical Mathematics. Springer; New York: 1991.

6. Connolly ML. Solvent-accessible surfaces of proteins and nucleic acids. Science. 1983; 221:709713. [PubMed: 6879170]

7. Bruccoleri RE, Novotny J, Davis ME, Sharp KA. Finite difference Poisson-Boltzmann electrostatic calculations: Increased accuracy achieved by harmonic dielectric smoothing and charge antialiasing. J Comput Chem. 1997; 18:268-276.

8. Im W, Beglov D, Roux B. Continuum solvation model: Computation of electrostatic forces from numerical solutions to the Poisson-Boltzmann equation. Comp Phys Commun. 1998; 111:59-75. 
9. Dong F, Olsen B, Baker NA. Computational methods for biomolecular electrostatics. Methods Cell Biol. 2008; 84:843-870. [PubMed: 17964951]

10. Li H, Robertson AD, Jensen JH. Very Fast Empirical Prediction and Rationalization of Protein pKa Values. Proteins. 2005; 61:704-721. [PubMed: 16231289]

11. Greenbaum, A. Iterative Methods for Solving Linear Systems (Frontiers in Applied Mathematics). SIAM; 1987.

12. Holst M, Saied F. Multigrid solution of the Poisson-Boltzmann equation. J Comput Chem. 1993; 14:105-113.

13. Hansen, JP.; McDonald, IR. Theory of Simple Liquids. 4th. Academic Press; Oxford: 2013. 
Matlab Program for Biomolecular Electrostatic Calculations

MPBEC

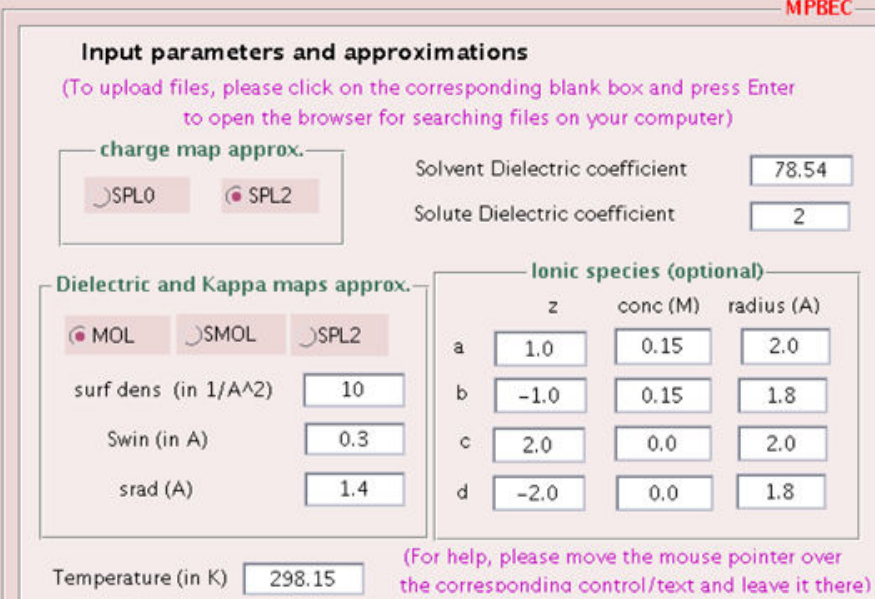

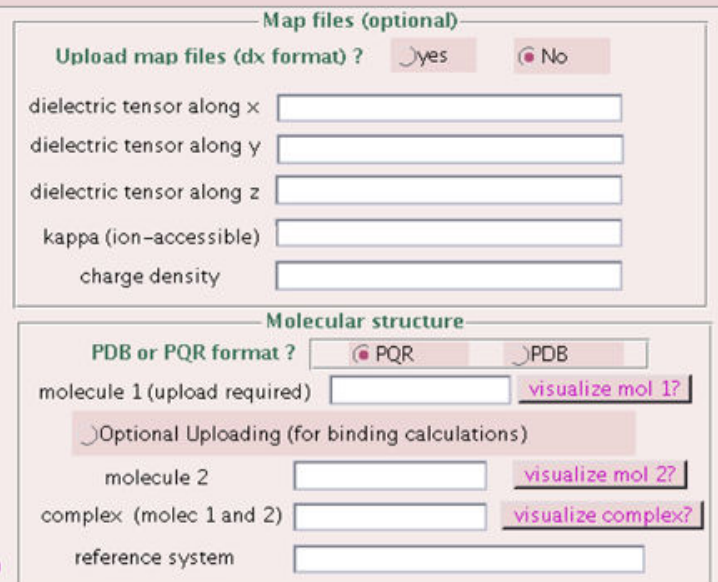

Write out files_Jcharge map 」kappa map_」dielectric maps $\nabla$ Mean Electrostatic Potential solution $\checkmark$ Electric Potential Surface plot Output Directory Click here and press enter to open a browser if you want to change the default output directory ( $/ \mathrm{MPBEC} / \mathrm{program} / \mathrm{src} / \mathrm{examples} / \mathrm{solutions})$

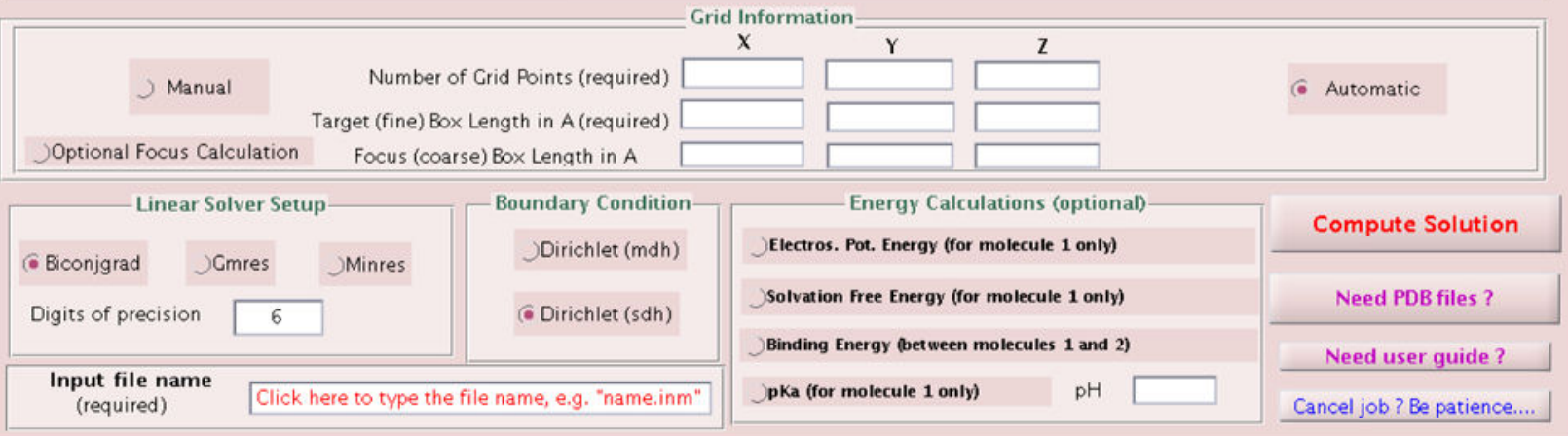

Figure 1.

Main Screen

Comput Phys Commun. Author manuscript; available in PMC 2016 February 25. 


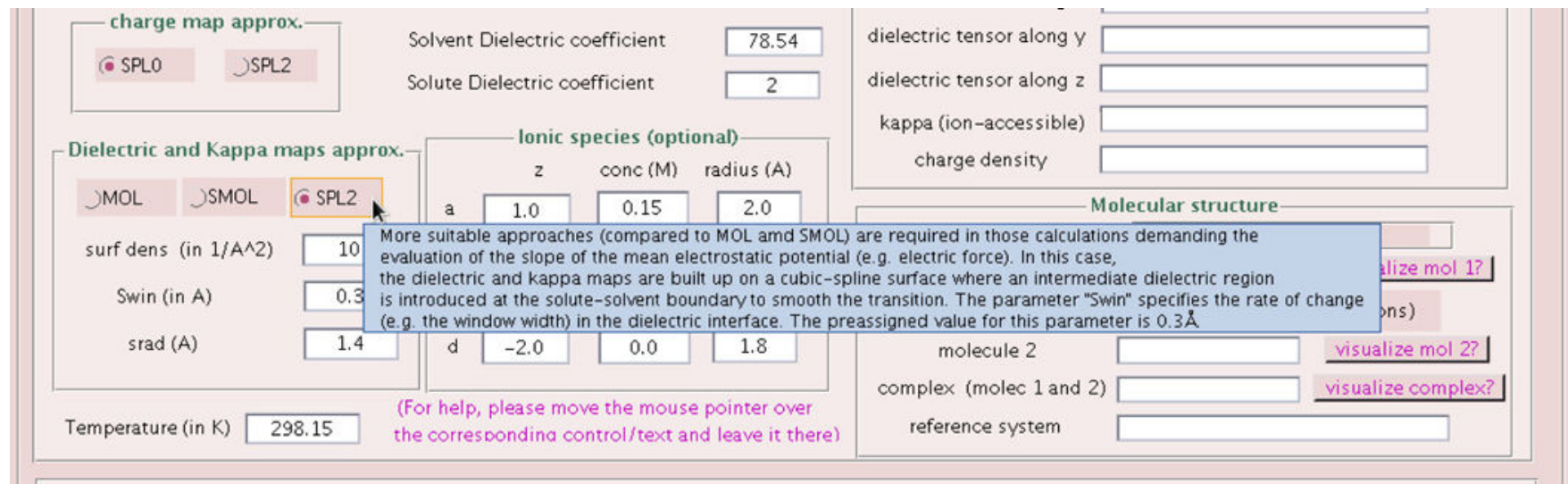

Figure 2.

Getting help messages 


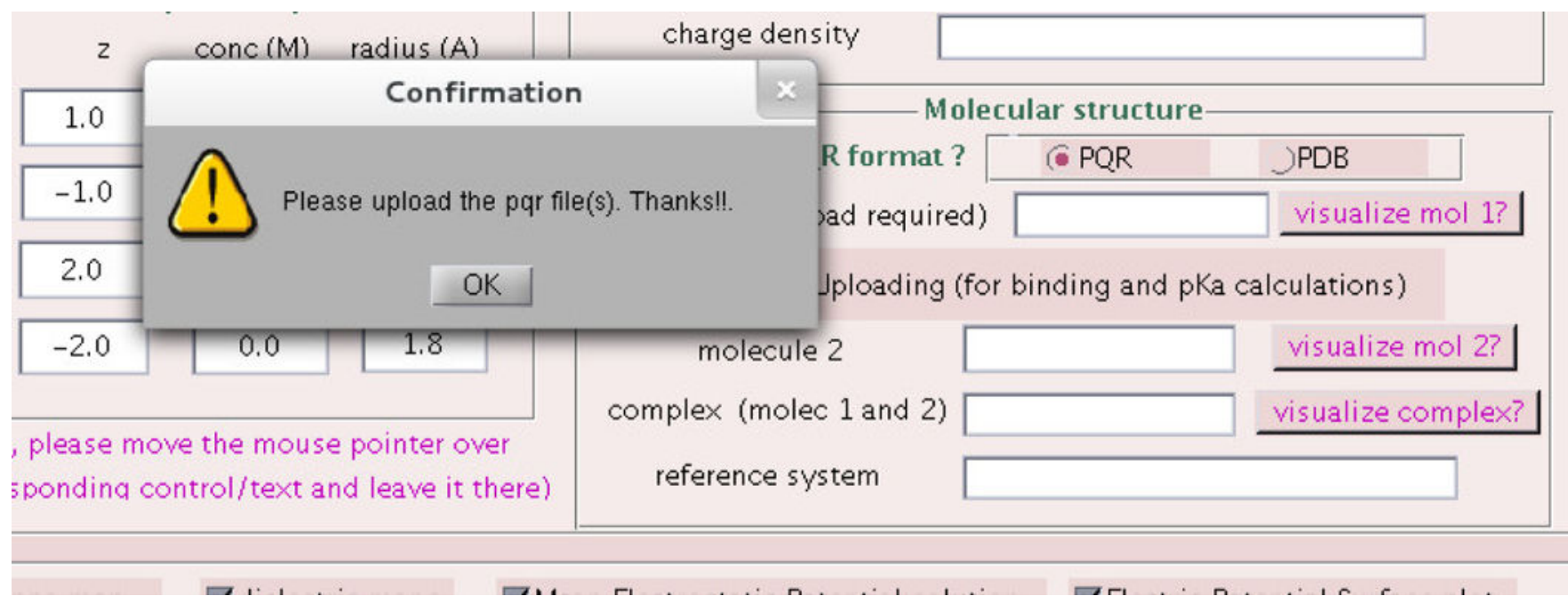

Figure 3.

Getting warning messages generated by the pre-test algorithm when an error in the input data is detected 


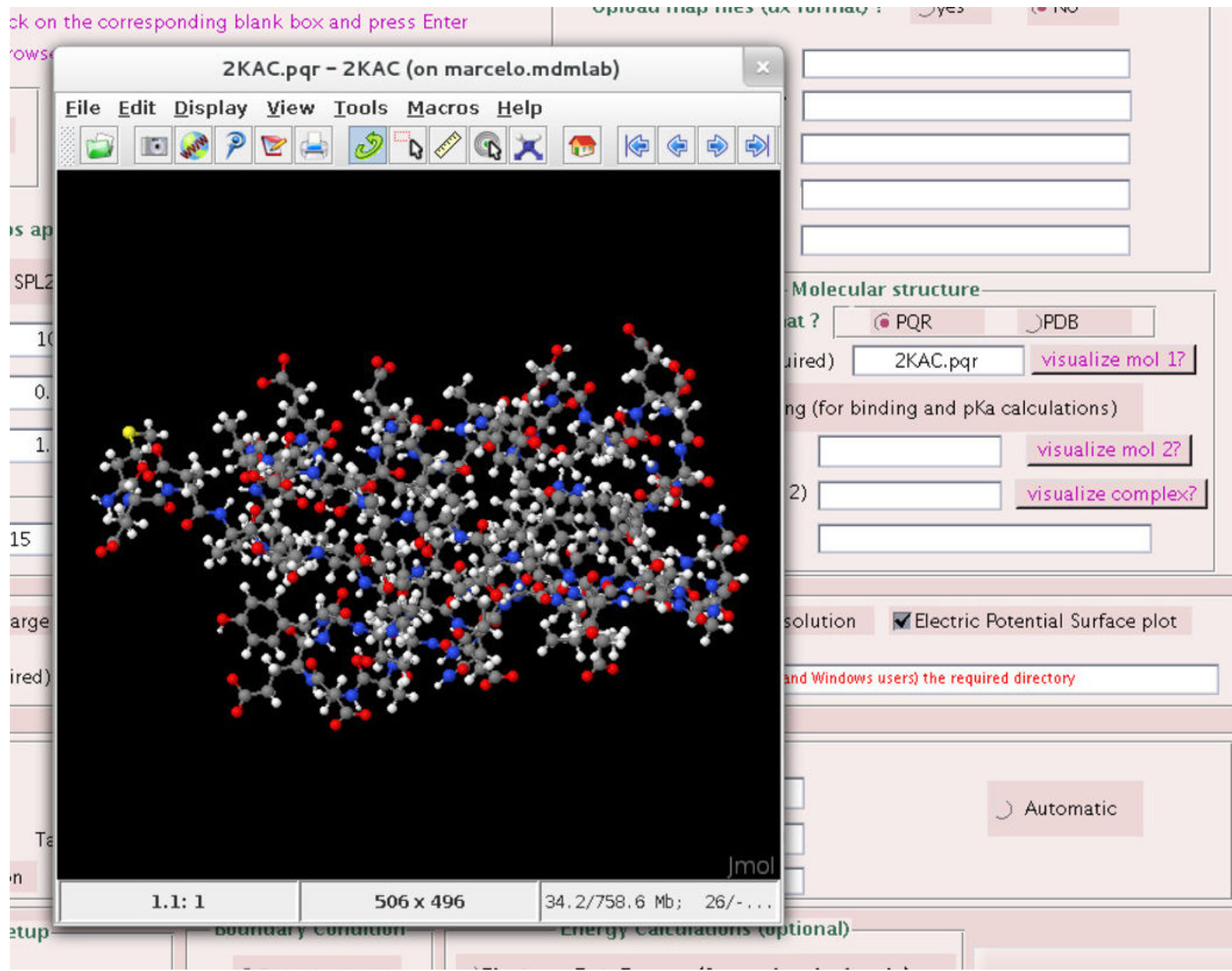

Figure 4.

Using the integrated Jmol application to visualize molecular structures 


\section{Input parameters and approximations}

'To upload files, please click on the corresponding blank box and press Enter to open the browser for searching files on your computer) - charge map approx.

JPLO $\quad$ SPL2

lielectric and Kappa maps app

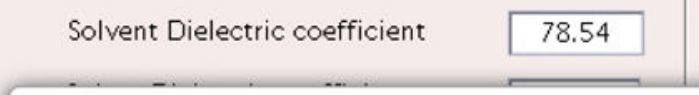

78.54 dielectric tensor along $x$ dielectric tensor along $y$

\begin{tabular}{|l|l} 
mapbsinput.pqr-mapbsinput (on marcelo.mdmlab) \\
File Edit Display View Iools Macros Help
\end{tabular}

$J \mathrm{MOL} \quad J \mathrm{SMOL} \quad \mathrm{SPL} 2$

surf dens (in 1/A^2)

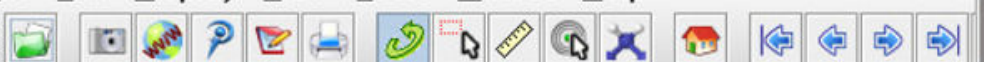

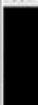

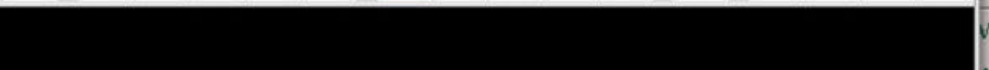

Map tiles (optional)

Upload map files (dx format)?

Jyes

\section{\begin{tabular}{l|c} 
Swin (in A) & 0.3 \\
$\operatorname{srad}(\mathrm{A})$ & 1.4
\end{tabular} \\ \begin{tabular}{l|c} 
Swin (in A) & 0.3 \\
$\operatorname{srad}(\mathrm{A})$ & 1.4
\end{tabular} \\ \begin{tabular}{ll|}
\hline emperature (in K) & 298.15 \\
\hline Urite out files $\quad \nabla$ charge $n$
\end{tabular}}

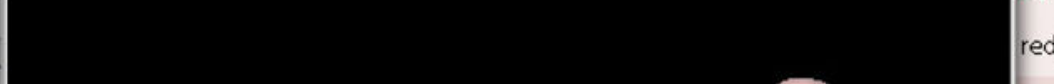

Molecular structure

G PQR

2KAC.pqr

(for binding and pKa calc

Output Directory (required)
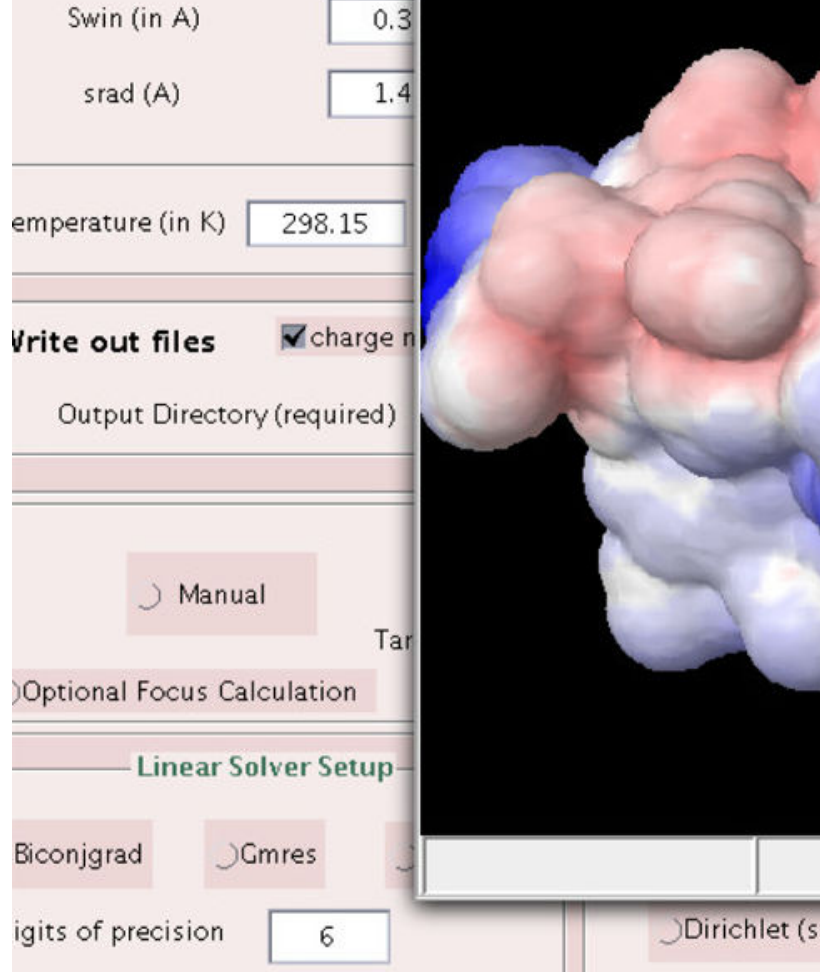

\section{Optional Focus Calculation}
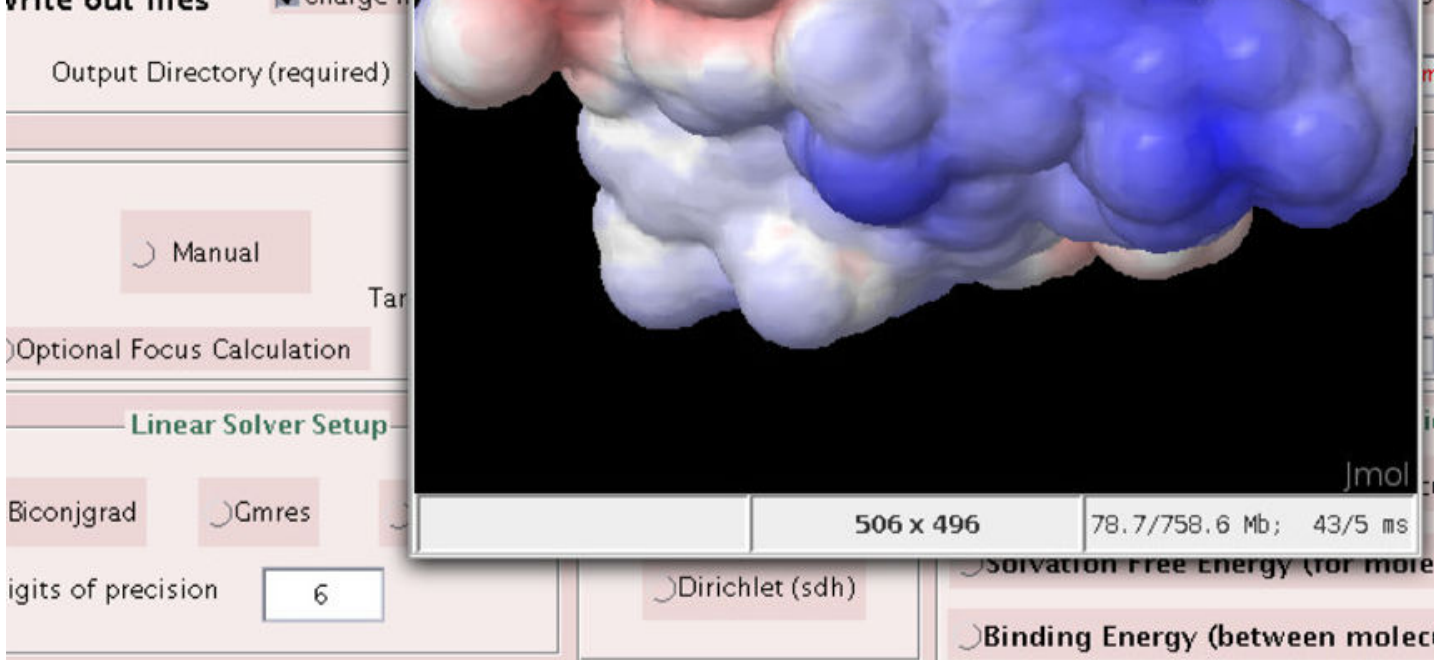

\section{Input file name}

(required)

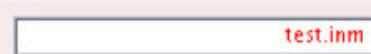

Dirichlet (sdh)

Binding Energy (between molecules 1 and 2)

pKa (for molecule 1 only) $\mathrm{pH}$

Figure 5.

Using the integrated Jmol application to visualize the solution of the mean electrostatic potential on the biomolecule surface. 
Figure 1

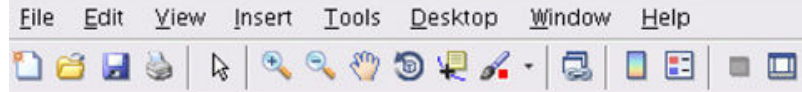

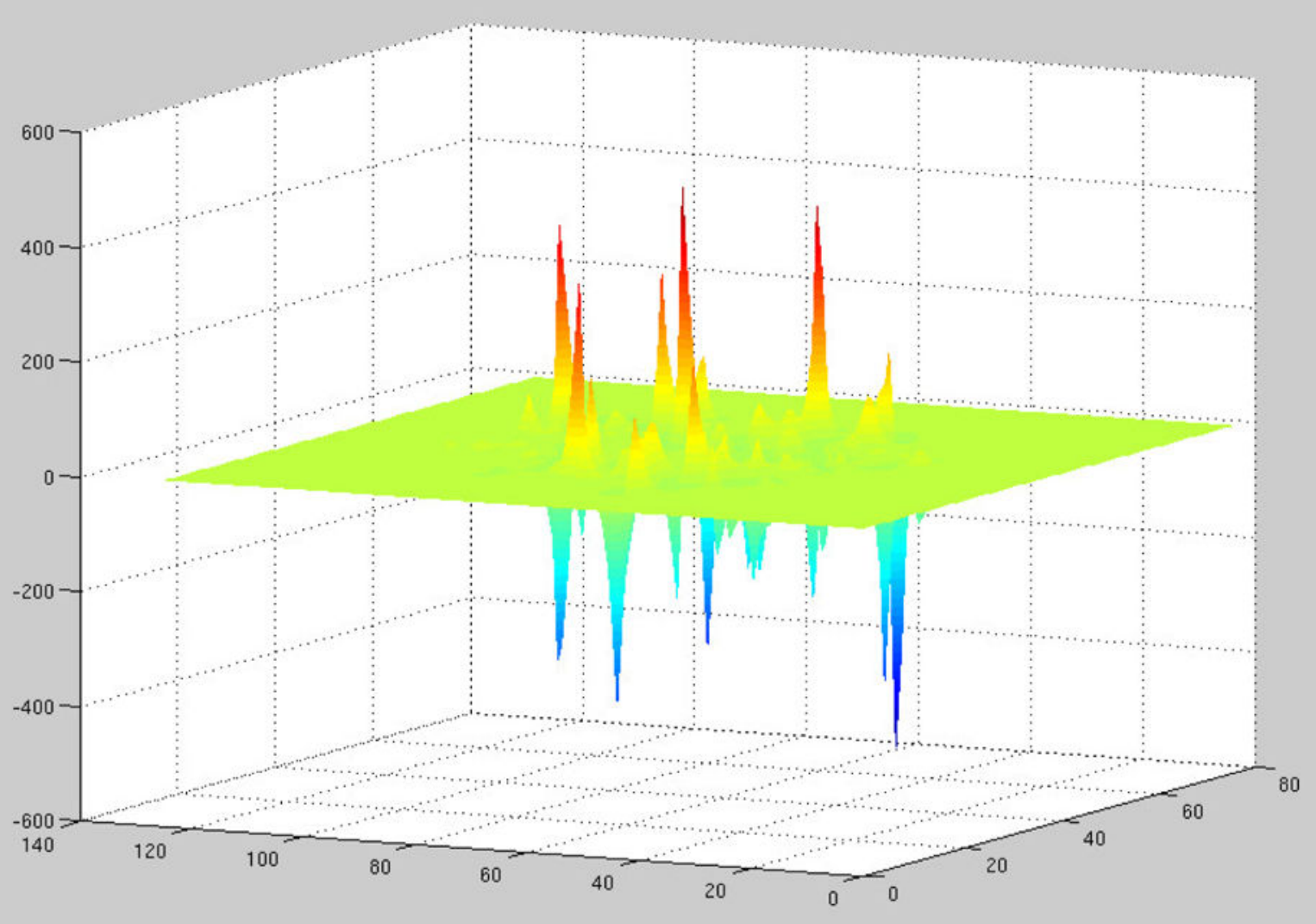


Figure 1

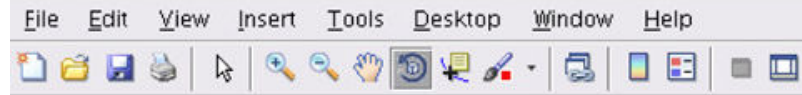

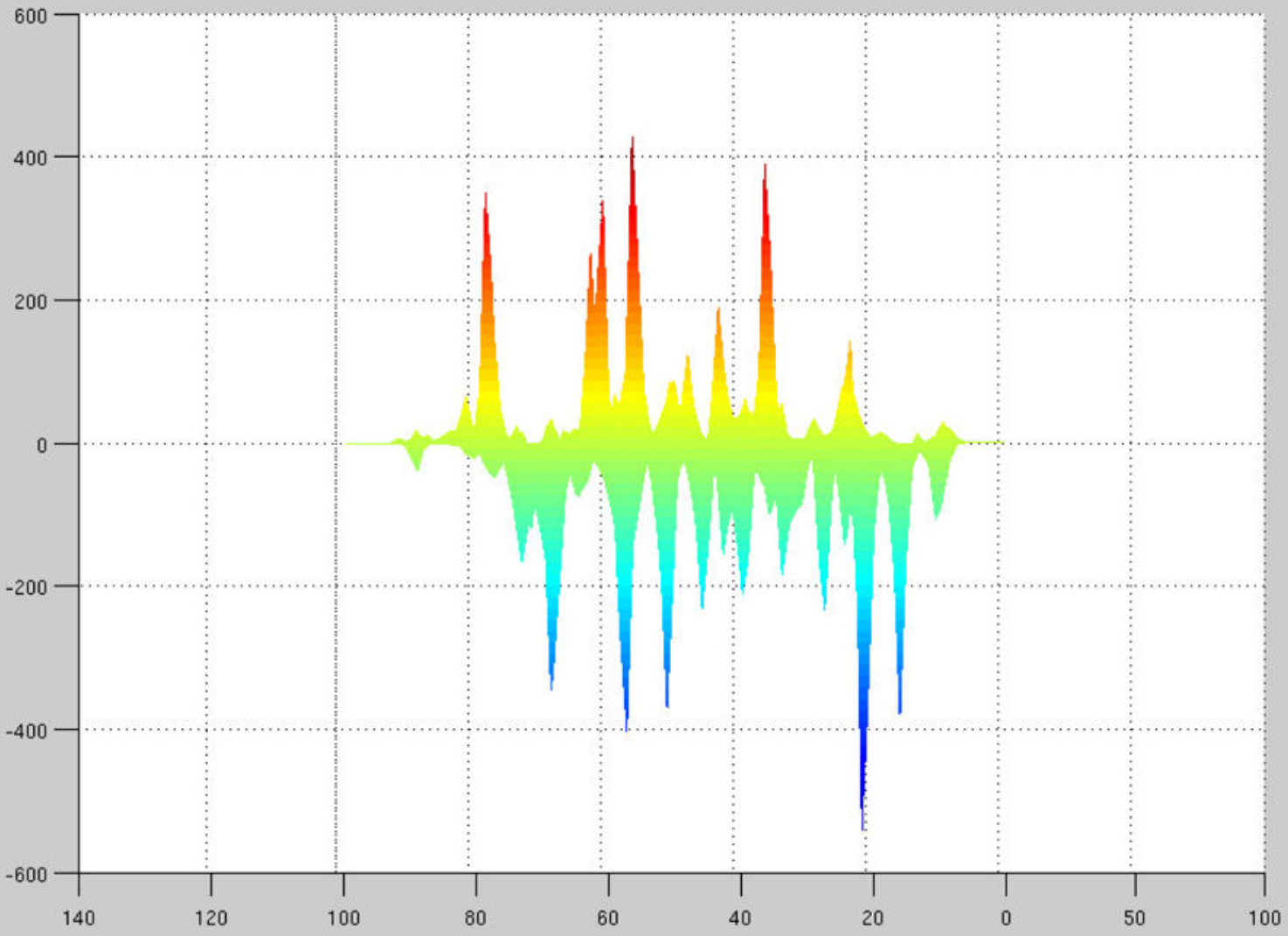

Figure 6.

Using Matlab tools to visualize the asymptotic behavior of the solution of the mean electrostatic potential. 


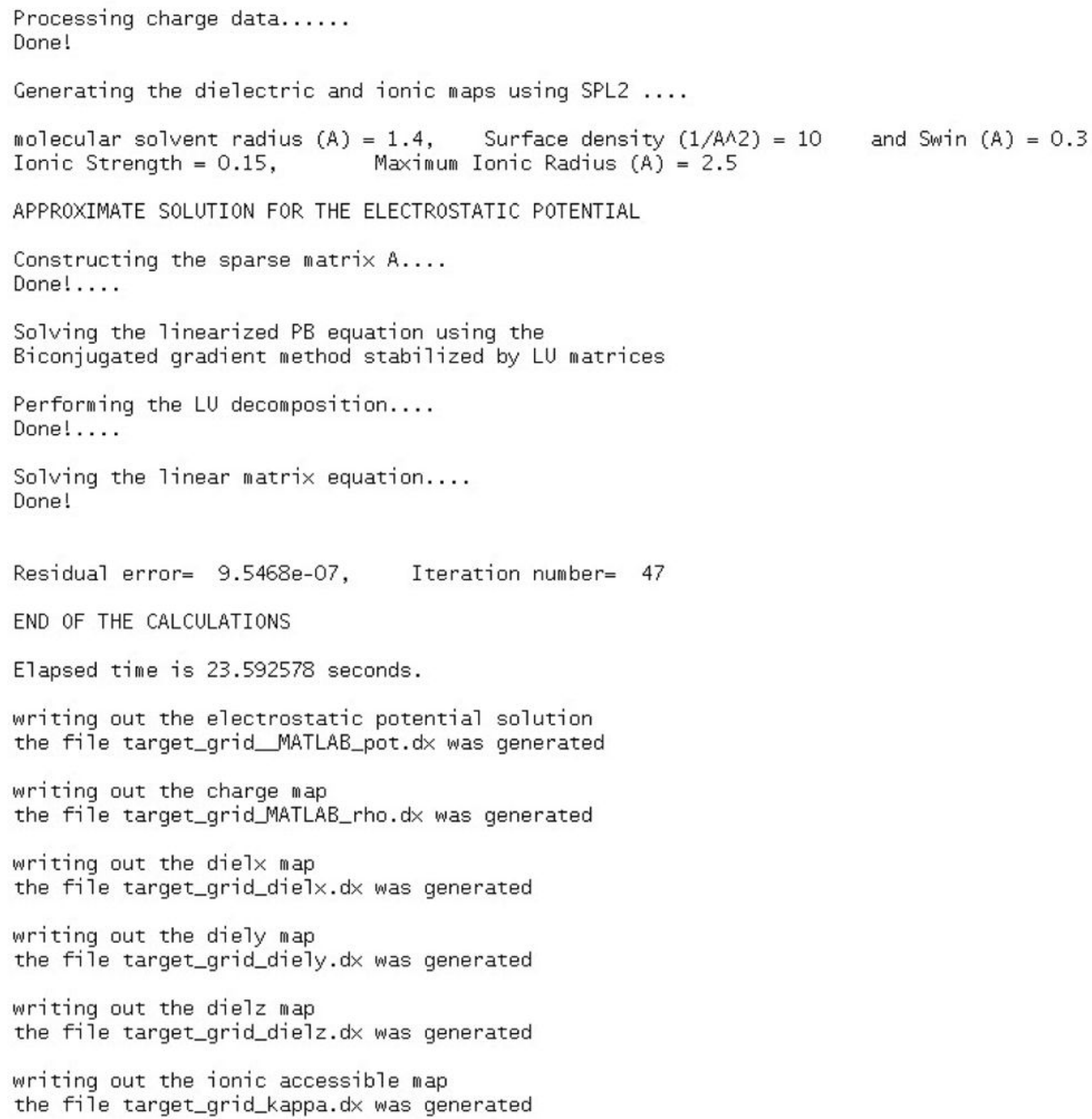




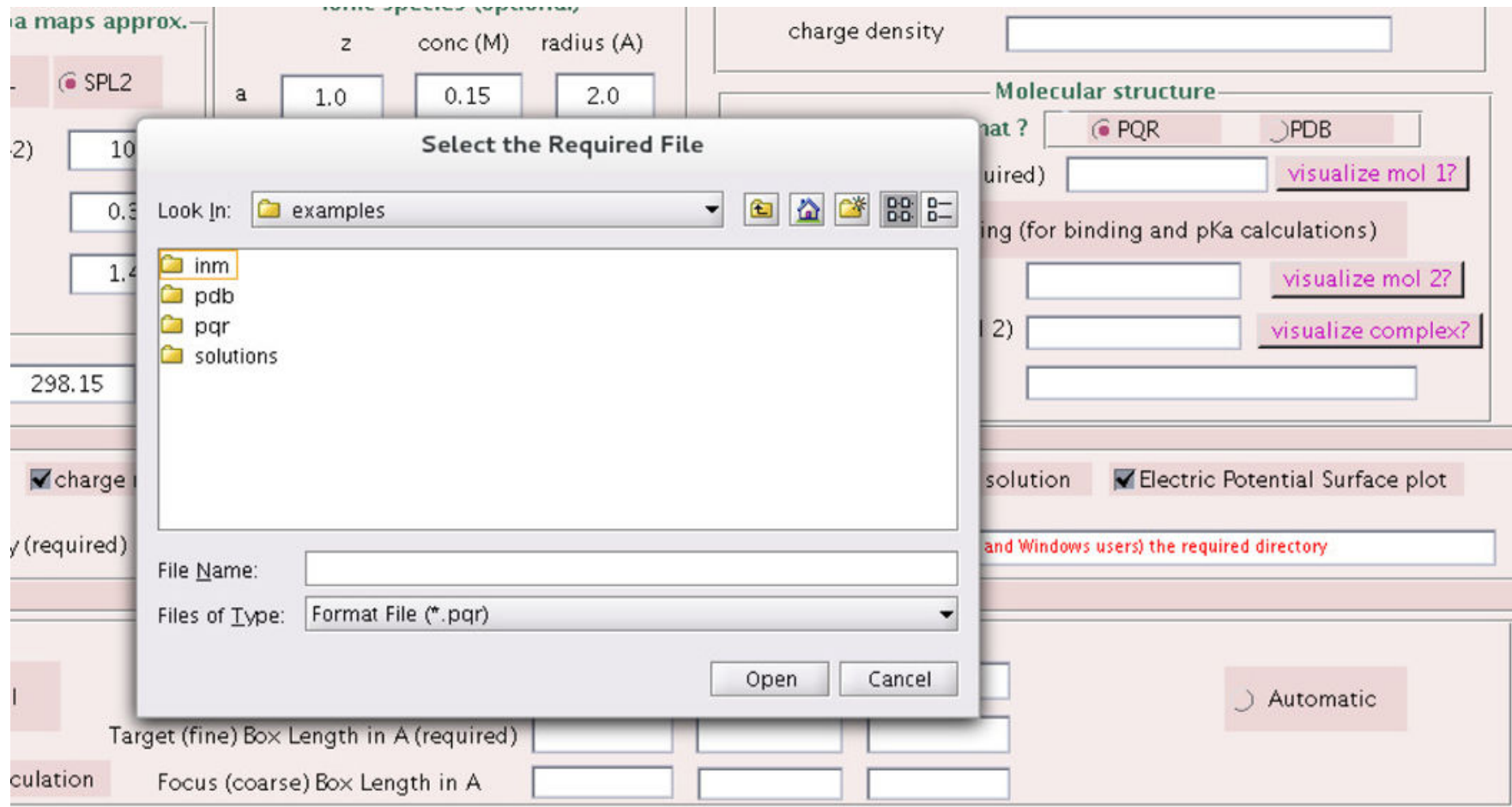

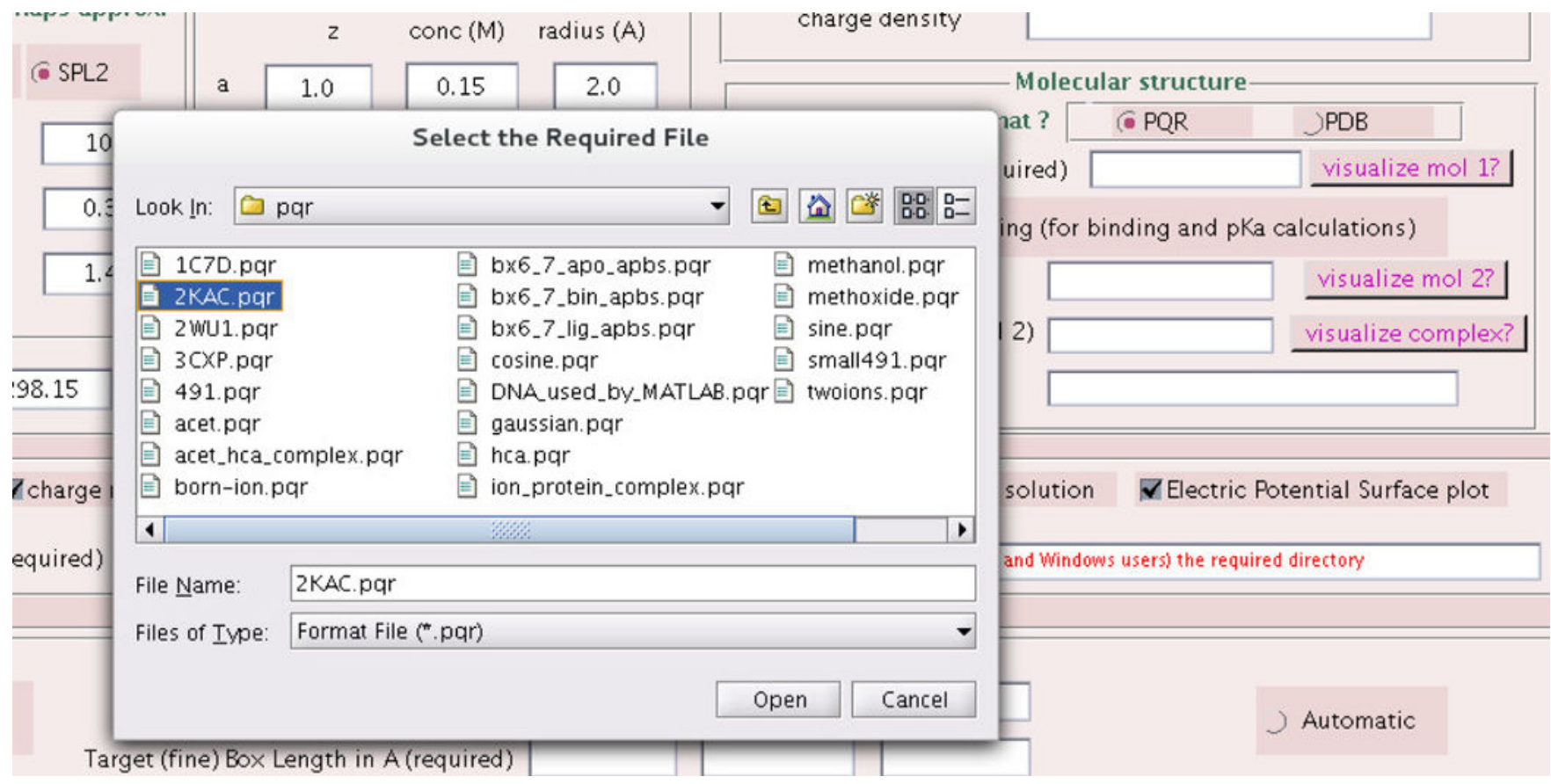

Figure 8.

Input files uploading 

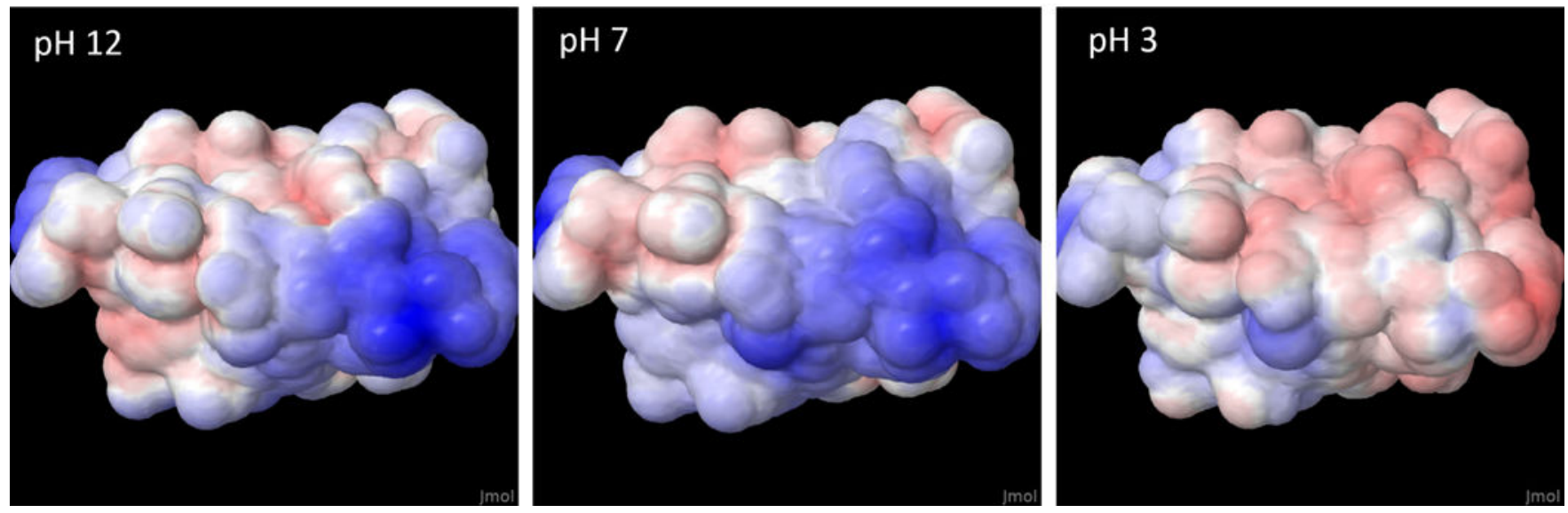

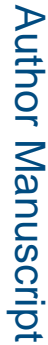

Figure 9.

Mean electrostatic surface potential for different $\mathrm{pH}$ values 
Table 1

Born ion model. Energy calculations for different number of grid points.

\begin{tabular}{|c|c|c|c|}
\hline Grid points $x=y=z$ & & $\operatorname{MPBEC}(\mathbf{k J} / \mathbf{m o l})$ & $\operatorname{APBS}(\mathrm{kJ} / \mathrm{mol})$ \\
\hline \multirow{3}{*}{97} & Solvated energy & 6387.46 & 6386.11 \\
\hline & Reference energy & 6618.70 & 6618.78 \\
\hline & Solvation energy & -231.24 & -232.67 \\
\hline \multirow{3}{*}{129} & Solvated energy & 8595.29 & 8595.04 \\
\hline & Reference energy & 8824.93 & 8825.04 \\
\hline & Solvation energy & -229.64 & -230.00 \\
\hline \multirow{3}{*}{161} & Solvated energy & 10801.5 & 10801.5 \\
\hline & Reference energy & 11031.2 & 11031.3 \\
\hline & Solvation energy & -229.65 & -229.78 \\
\hline \multirow{3}{*}{193} & Solvated energy & 13007.7 & 13007.7 \\
\hline & Reference energy & 13237.4 & 13237.6 \\
\hline & Solvation energy & -229.67 & -229.87 \\
\hline \multirow{3}{*}{257} & Solvated energy & 17420.6 & 17420.7 \\
\hline & Reference energy & 17649.9 & 17650.1 \\
\hline & Solvation energy & -229.22 & -229.42 \\
\hline \multirow{3}{*}{353} & Solvated energy & 24039.7 & 24039.7 \\
\hline & Reference energy & 24268.6 & 24268.9 \\
\hline & Solvation energy & -228.89 & -229.16 \\
\hline
\end{tabular}

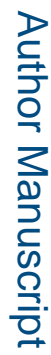


Table 2

Born ion model. Energy calculations for different options of charge map (chm) and dielectric and kappa maps (dkm).

\begin{tabular}{|c|c|c|c|c|}
\hline chm & dkm & & $\operatorname{MPBEC}(\mathbf{k J} / \mathbf{m o l})$ & $\operatorname{APBS}(\mathrm{kJ} / \mathrm{mol})$ \\
\hline \multirow{9}{*}{$\operatorname{spl} 2$} & \multirow{3}{*}{ mol } & Solvated energy & 5352.48 & 5352.35 \\
\hline & & Reference energy & 5582.15 & 5582.22 \\
\hline & & Solvation energy & -229.67 & -229.87 \\
\hline & \multirow{3}{*}{ smol } & Solvated energy & 5353.38 & 5353.13 \\
\hline & & Reference energy & 5582.15 & 5582.22 \\
\hline & & Solvation energy & -228.77 & -229.09 \\
\hline & \multirow{3}{*}{$\mathrm{spl} 2$} & Solvated energy & 5333.17 & 5333.23 \\
\hline & & Reference energy & 5582.15 & 5582.22 \\
\hline & & Solvation energy & -248.98 & -248.98 \\
\hline \multirow{9}{*}{ splo } & \multirow{3}{*}{$\mathrm{mol}$} & Solvated energy & 13007.7 & 13007.7 \\
\hline & & Reference energy & 13237.4 & 13237.6 \\
\hline & & Solvation energy & -229.67 & -229.87 \\
\hline & \multirow{3}{*}{ smol } & Solvated energy & 13008.6 & 12988.6 \\
\hline & & Reference energy & 13237.4 & 13237.6 \\
\hline & & Solvation energy & -228.77 & -229.09 \\
\hline & \multirow{3}{*}{$\mathrm{spl} 2$} & Solvated energy & 12988.4 & 12988.6 \\
\hline & & Reference energy & 13237.4 & 13237.6 \\
\hline & & Solvation energy & -248.98 & -248.98 \\
\hline
\end{tabular}

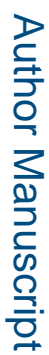

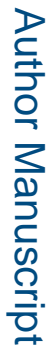

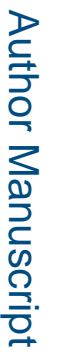


Table 3

Methanol model. Energy calculations for different charge map (chm) options and electrolyte concentrations.

\begin{tabular}{|c|c|c|c|c|}
\hline Ionic strength & chm & & $\operatorname{MPBEC}(\mathrm{kJ} / \mathrm{mol})$ & $\operatorname{APBS}(\mathrm{kJ} / \mathrm{mol})$ \\
\hline \multirow{6}{*}{$0 \mathrm{M}$} & \multirow{3}{*}{ spl0 } & Solvated energy & 2681.14 & 2681.17 \\
\hline & & Reference energy & 2706.61 & 2706.64 \\
\hline & & Solvation energy & -25.47 & -25.47 \\
\hline & \multirow{3}{*}{$\mathrm{spl} 2$} & Solvated energy & 1648.74 & 1648.76 \\
\hline & & Reference energy & 1674.05 & 1674.07 \\
\hline & & Solvation energy & -25.31 & -25.31 \\
\hline \multirow{6}{*}{$0.05 \mathrm{M}$} & \multirow{3}{*}{ spl0 } & Solvated energy & 2681.14 & 2681.17 \\
\hline & & Reference energy & 2706.37 & 2706.29 \\
\hline & & Solvation energy & -25.23 & -25.12 \\
\hline & \multirow{3}{*}{$\mathrm{spl} 2$} & Solvated energy & 1648.74 & 1648.76 \\
\hline & & Reference energy & 1673.81 & 1673.72 \\
\hline & & Solvation energy & -25.07 & -24.96 \\
\hline \multirow{6}{*}{$0.5 \mathrm{M}$} & \multirow{3}{*}{ spl0 } & Solvated energy & 2681.14 & 2681.16 \\
\hline & & Reference energy & 2705.93 & 2705.83 \\
\hline & & Solvation energy & -24.80 & -24.67 \\
\hline & \multirow{3}{*}{$\mathrm{spl} 2$} & Solvated energy & 1648.74 & 1648.75 \\
\hline & & Reference energy & 1673.37 & 1673.25 \\
\hline & & Solvation energy & -24.63 & -24.50 \\
\hline
\end{tabular}

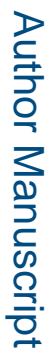


Table 4

Methoxide (Ionized Methanol) model. Energy calculations for different charge map (chm) options and electrolyte concentrations.

\begin{tabular}{|c|c|c|c|c|}
\hline Ionic strength & chm & & $\operatorname{MPBEC}(\mathbf{k J} / \mathbf{m o l})$ & $\operatorname{APBS}(\mathbf{k J} / \mathrm{mol})$ \\
\hline \multirow{6}{*}{$0 \mathrm{M}$} & \multirow{3}{*}{ spl0 } & Solvated energy & 5633.92 & 5633.99 \\
\hline & & Reference energy & 5834.53 & 5834.60 \\
\hline & & Solvation energy & -200.61 & -200.61 \\
\hline & \multirow{3}{*}{$\mathrm{spl} 2$} & Solvated energy & 2485.68 & 2485.71 \\
\hline & & Reference energy & 2686.32 & 2686.35 \\
\hline & & Solvation energy & -200.64 & -200.64 \\
\hline \multirow{6}{*}{$0.05 \mathrm{M}$} & \multirow{3}{*}{ spl0 } & Solvated energy & 5633.56 & 5633.48 \\
\hline & & Reference energy & 5784.86 & 5777.48 \\
\hline & & Solvation energy & -151.30 & -144.00 \\
\hline & \multirow{3}{*}{$\mathrm{spl} 2$} & Solvated energy & 2485.32 & 2485.20 \\
\hline & & Reference energy & 2636.65 & 2629.23 \\
\hline & & Solvation energy & -151.33 & -144.03 \\
\hline \multirow{6}{*}{$0.5 \mathrm{M}$} & \multirow{3}{*}{ sp10 } & Solvated energy & 5633.42 & 5632.90 \\
\hline & & Reference energy & 5762.95 & 5758.42 \\
\hline & & Solvation energy & -129.53 & -125.53 \\
\hline & \multirow{3}{*}{ spl2 } & Solvated energy & 2485.18 & 2484.62 \\
\hline & & Reference energy & 2614.75 & 2610.18 \\
\hline & & Solvation energy & -129.56 & -125.56 \\
\hline
\end{tabular}

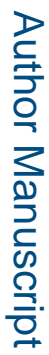


Table 5

Hca-Acetazolamine complex model. Binding energy calculation using focusing boundary condition

\begin{tabular}{|c|c|c|c|c|}
\hline Molecule & & Grid & $\operatorname{MPBEC}(\mathbf{k J} / \mathbf{m o l})$ & $\operatorname{APBS}(\mathbf{k J} / \mathbf{m o l})$ \\
\hline \multirow{2}{*}{ Hca } & \multirow{6}{*}{ Electrostatic potential energy } & Coarse & 127649.66 & 127651.22 \\
\hline & & Fine & 298150.07 & 298150.51 \\
\hline \multirow{2}{*}{ Acetazolamine } & & Coarse & 1147.24 & 1147.25 \\
\hline & & Fine & 2660.16 & 2660.19 \\
\hline \multirow{3}{*}{ Hca-Acetazolamine } & & Coarse & 128787.93 & 128789.56 \\
\hline & & Fine & 300749.95 & 300754.49 \\
\hline & Binding energy & & -60.29 & -60.22 \\
\hline
\end{tabular}

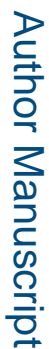

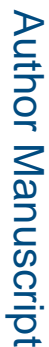

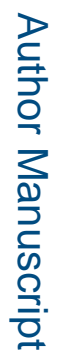

Comput Phys Commun. Author manuscript; available in PMC 2016 February 25. 
Table 6

1d7h - DMSO complex model. Binding energy calculation using focusing boundary conditions.

\begin{tabular}{|c|c|c|c|c|}
\hline Molecule & & Grid & $\operatorname{MPBEC}(\mathbf{k J} / \mathbf{m o l})$ & $\operatorname{APBS}(\mathbf{k J} / \mathbf{m o l})$ \\
\hline \multirow{2}{*}{$1 \mathrm{D} 7 \mathrm{H}$} & \multirow{6}{*}{ Electrostatic potential energy } & Coarse & 70246.2034 & 70246.8009 \\
\hline & & Fine & 166946.98 & 166948.75 \\
\hline \multirow{2}{*}{ DMSO } & & Coarse & 145.8681 & 145.8687 \\
\hline & & Fine & 310.5369 & 310.5395 \\
\hline \multirow{3}{*}{ 1D7H-DMSO } & & Coarse & 70394.73 & 70395.33 \\
\hline & & Fine & 167258.31 & 167260.10 \\
\hline & Binding energy & & 0.7968 & 0.7997 \\
\hline
\end{tabular}




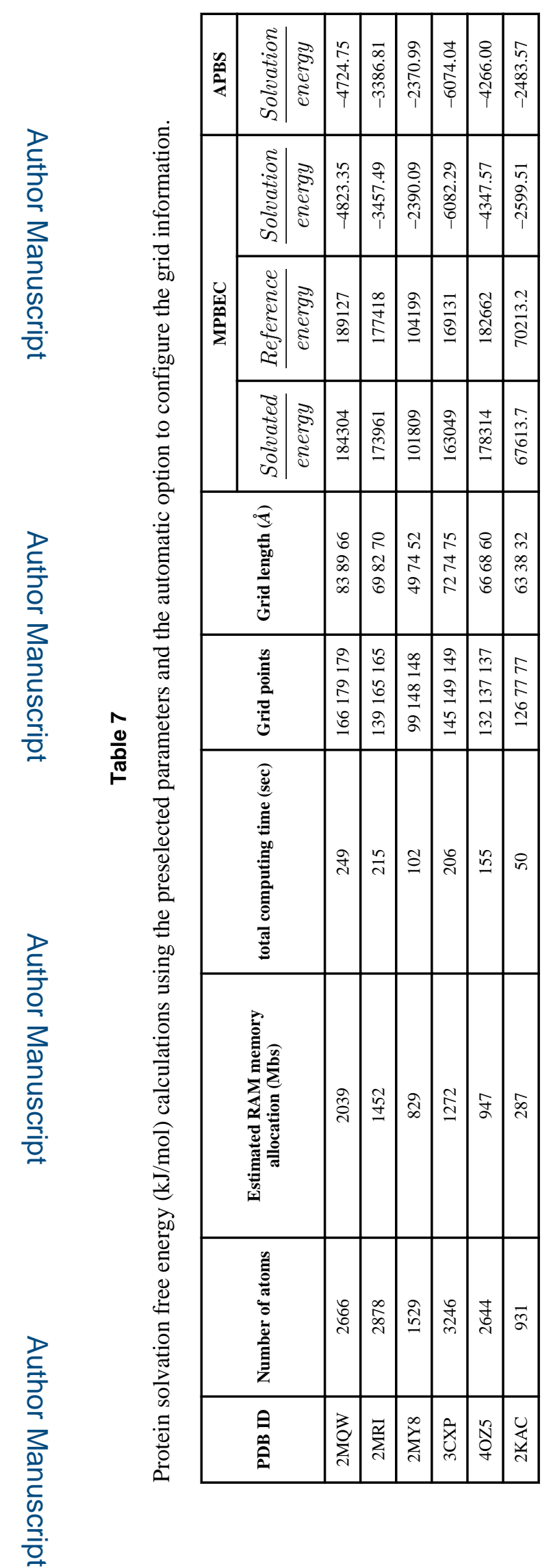

Comput Phys Commun. Author manuscript; available in PMC 2016 February 25. 